\title{
Orthogonal Learning-Based Gray Wolf Optimizer For Identifying The Uncertain Parameters of Various Photovoltaic Models
}

\section{Felix Joseph Xavier}

Bule Hora University

\section{A.Pradeep}

Noorul Islam Centre For Higher Education

\section{A.Anbarasan}

P.V.P Siddhartha Institute Of Technology

C.Kumar ( $\square$ ckumarme81@gmail.com )

M. Kumarasamy College of Engineering https://orcid.org/0000-0002-1132-4794

\section{Research Article}

Keywords: Convergence, Gray Wolf Optimizer (GWO), Photovoltaic parameters, Optimization, Orthogonal learning

Posted Date: May 11th, 2021

DOl: https://doi.org/10.21203/rs.3.rs-234955/v1

License: (c) (i) This work is licensed under a Creative Commons Attribution 4.0 International License. Read Full License

Version of Record: A version of this preprint was published at Optik on December 1st, 2021. See the published version at https://doi.org/10.1016/j.ijleo.2021.167973. 


\title{
Orthogonal Learning-Based Gray Wolf Optimizer for Identifying the Uncertain Parameters of Various Photovoltaic Models
}

\author{
Felix Joseph Xavier ${ }^{1}$, A.Pradeep ${ }^{2}$, A.Anbarasan ${ }^{3}$, C.Kumar ${ }^{4 *}$ \\ ${ }^{1}$ Assistant Professor, Electrical and Computer Engineering, Bule Hora University, Ethiopia. \\ ${ }^{2}$ Research Scholar, EEE, Noorul Izlam Center for Higher Education, Kanyakumari. \\ ${ }^{3}$ Associate Professor, Electrical and Electronics Engineering, P.V.P Siddhartha Institute of Technology, Vijayawada. \\ ${ }^{4 *}$ Professor, Electrical and Electronics Engineering, M.Kumarasamy College of Engineering, Karur. \\ *Corresponding Author: ckumarme81@gmail.com
}

\begin{abstract}
Determining the optimal parameters for the photovoltaic system (PV) model is essential during the design, evolution, development, estimation, and PV systems analysis. Therefore, it is crucial for the proper advancement of the best parameters of the PV models based on modern computational techniques. Thus, this work suggests a new Orthogonal-LearningBased Gray Wolf Optimizer (OLBGWO) through a local exploration for estimating the unknown variables of PV cell models. The exploitation and exploration capability of the basic Gray Wolf Optimizer (GWO) is improved by the orthogonal-learning-based (OLB) approach, and this arrangement promotes a highly reliable equilibrium between the exploitation and exploration levels of the algorithm. In OLBGWO, the OLB strategy is used to find the best solution for the poor populations and directs the population to review the potential search area during the iterative process. Also, an exponential decay function is employed to decrease the value of vector $a$ in GWO. The developed algorithm is directly applied to the parameter identification problem of the PV system. The proposed OLBGWO algorithm estimates the unknown parameters of the single-diode model (SDM), double-diode model (DDM), and PV module model. The performance of the OLBGWO is compared with other competitive algorithms to prove its superiority. The simulation results prove that the OLBGWO algorithm can achieve high solution accuracy with high convergence speed.
\end{abstract}

Key Words - Convergence; Gray Wolf Optimizer (GWO); Photovoltaic parameters; Optimization; Orthogonal learning

\section{Introduction}

Renewable energy can become a growing technology because of fossil fuel usage that can contribute to catastrophe and atmospheric pollution and change energy usage configurations 
from fossil fuel to green energy [1,2]. Society has demanded renewable energy to be used in water, wind, solar, and several other fields, where solar energy has tremendous potential due to its abundance of supplies and environment responsive. Because of the effective use of solar power in producing electric power, solar photovoltaic (PV) systems are much in demand, and developments are continuing to grow [3,4]. However, there are some detrimental aspects to the performance of the PV systems, such as direct exposure of the panel to the outside and inadequate productivity of PV panels [5,6]. Therefore, it is important to identify the practical efficiency of PV modules to plan effectively, optimize, estimate, control, and simulate PV systems. The actual model is used as per the current and voltage samples collected to accomplish this purpose [7]. The PV model can be constructed using the mathematical model, and the internal parameters can be defined [8,9]. The researchers have reported numerous PV models, such as the Single-Diode Model (SDM), Double-Diode Model (DDM), and Three-Diode Model (TDM), and out of which the SDM and DDM are preferred models for PV systems. Besides, the performance of PV models is based on uncertain internal parameters [10]. It is challenging to evaluate all the unknown parameters and keep them stable due to aging, deterioration, and unstable working states. It is critical to estimate, emulate, design, and optimize the PV systems without defining the above-said parameters. Therefore, attention is drawn to the performance of swarm-based optimization algorithms in estimating variables in PV systems [11,12].

To construct a computation model for photovoltaic systems, an appropriate objective function needs to be used. Fitness is the multimodal function due to noise in the collected current and voltage samples, and it is not easy to deal with that to find viable solutions $[13,14]$. The researchers spent countless days dealing with this issue and creating several different techniques. Previously, deterministic approaches were used by several researchers to mitigate such challenges. For example, the exact method, 5-point analytical method, Newton method [15], and curve-fitting method [16], are used to address the same problem. Several gradient computations are required for deterministic methods and perform well when performing a local search. The authors of [17] reported an analytical technique to identify the parameters of the PV cell. However, the authors have not discussed the various PV models. In comparison, it is quickly struck at the local optimum. This inactivity problem would prevent the algorithm from optimizing solutions' quality on further reiterations [18,19]. Such approaches, with the initial stage, are rigid. Thus, the choice of initial points has a significant influence on the speed of 
convergence and the reliability of the solution of such methods. The researchers ought to have an objective function with convex and distinguishable features to use these methods; these restrictions can restrict the application of these techniques. Evolutionary approaches have discovered their effectiveness and outcomes relative to conventional methods without such strict limitations [20,21]. In recent years, specific optimizers and their enhancement variants have also been reported to define the PV system variables.

To identify the model parameters under various operating conditions, a penalty-based differential evolution was reported in [22]. An enhanced adaptive differential evolutionary (DE) algorithm with a rigid design focused on objective value was discussed to identify variables of solar panels [23]. To identify the model parameters, a chaotic particle-swarm-optimization (PSO) technique was suggested. The chaotic search [24] improves the global search and convergence ability. To define PV cell parameters, simulated annealing (SA) was used, and then the SDM, DDM, and PV module models have been utilized to check the efficiency of SA [25]. The authors of [26] merged SA with Levenberg-Marquardt to address problems with extracting parameters. To refine the variables of the three models described, the researchers of [27] used a Moth Flame Optimizer (MFO). To mimic the PV cell characteristics, the authors of [28] used a bacterial foraging algorithm. The authors of [29] implemented the artificial bee colony to extract the parameters of the PV cells. The artificial bee swarm optimization [30] and harmony search algorithm [31] have been introduced for parameter extraction of SDM and DDM. The authors of [32] constructed five different mutant bacterial foraging algorithms for SDM and DDM parameter estimation. For parameter identification of various models, the authors of [33] integrated the generalized opposition-learning and Nelder-Mead simplex method into the flower pollination algorithm. To estimate the variables of SDM and DDM, the authors of [34] developed mixed cooperative swarm optimization algorithms. The Salp Swarm Algorithm (SSA) was used by the authors of [35] to optimize the DDM parameters. For static and dynamic parameter identification of the PV models, the authors of [36] suggested a chaotic widespread learning PSO. The authors of [37] suggested a chaotic Jaya algorithm for finding the unknown variables of the SDM, DDM, and PV module model, including the commercial PV modules. The authors of [38] suggested hybrid Gray Wolf Optimizer (GWO) to extract the PV cell parameters; however, the basic version of GWO is positively stuck at local optima when it solves multimodal optimization problems. The authors of [39,40] proposed metaphorless and stochastic algorithms, such as the Rao algorithm 
[39] and Slime Mould Algorithm (SMA) [40] for extracting the uncertain parameters of various PV models. However, commercial PV modules are not analyzed. The authors of [41] reported political optimizer algorithms for estimating the PV cell parameters of SDM. However, the authors fail to describe the DDM and other commercial PV module model. To get the optimal parameters of a TDM, the authors of [42] used the sunflower optimization algorithm.

Although the metaheuristics and their alternatives are better than the deterministic approaches on the solution quality and the convergence rate, they have inherent drawbacks: the first is that the efficiency requires to be further enhanced on both the quality of the solution and the speed of convergence; the latter is that they have been developed to solve the particular problems of optimization, the inadequate portability limits the other practical problems. To get the best variables of PV models, the authors of [43] reported a reinforced moth search technique to define the best triple-junction PV module parameters in which the disruptor operator would increase the basic moth-search algorithm diversity. The authors of [44] implemented the opposition-basedlearning (OBL) and Nelder-Mead simplex techniques into the sine-cosine algorithm (SCA). The authors of [45] suggested the MFO with orthogonal Nelder-Mead concept for solar PV parameter identification optimization problem. The researchers of [46] merged the interior search algorithm with PSO to handle the parameter estimation of PV cell/module. Therefore, the authors of [38] suggest hybrid version GWO, i.e., by combining the features of PSO and GWO to identify the parameters of the PV cell. However, the convergence speed of the hybrid algorithm is relatively very high. The authors of [47] suggested Harris Hawks Optimizer (HHO) integrated by means of combining the OBL and chaotic local search for model parameter identification. The researchers of [23] reported an electromagnetism algorithm to obtain the unknown variables of the SDM of the PV panel.

The GWO algorithm for addressing parameter optimization frameworks and practical engineering issues was created by Mirjalili [48]. The GWO algorithm has been extended to several fields because it was developed and implemented to address different engineering practices. The GWO algorithm has been updated to be in line with the search space of diverse problems because of the difficulty of real-world optimization problems. The following are the possibilities to improve the GWO algorithm: Firstly, the update mechanism of the GWO is modified. Changes are made in the update stage because the GWO has some limitations to real- 
world engineering problems. The changes are possible by introducing an update mechanism, new operators, encoding schemes, and structural change of the population and hierarchy [49]. Latter, improvements have been made to enhance GWO by introducing chaotic concepts or levy flight concepts. To empower the discovery GWO algorithm, further enhanced versions apply the principle of hybridization. The hybrid versions are possible by integrating PSO, DE, etc., with the GWO algorithm. Another possible modification is introducing the crowding distance and non-dominated sorting approaches to convert the GWO into multiobjective GWO to handle large-scale optimization problems [50]. As per the No-Free-Lunch theorem, one algorithm might not be suitable for all types of engineering problems [51]. Therefore, this paper proposes a new update mechanism called orthogonal-learning-based strategy to improve the solution accuracy and convergence speed by considering the above-all facts. GWO's first version deals with the deterioration of exploratory tendencies and the intensity of convergence. In this paper, an improved GWO to find variables of various PV models is incorporated with the OLB strategy. The key contributions of this study are given as follows:

- A new algorithm called the OLBGWO algorithm is proposed by integrating the concept of orthogonal-learning with the GWO algorithm.

- A better candidate solution for GWO is created using OLB, which directs the weaker populations to acceptable search areas to increase the exploitation and exploration abilities. Besides, OLB assists the weaker populations in leaping out of the local optimum and speeding up the exploitation and exploration abilities of the GWO.

- The OLB mechanism leads to the necessary balance between exploitation and exploration.

- In addition to OLB, the exploration phase of the GWO is also improved by employing the exponential decay function to decrease the value of vector $a$ in GWO.

- The OLBGWO algorithm performance is demonstrated by the experimental results and comparisons with other competitive algorithms.

This research is arranged as follows: The description of the problem is provided in Section 2. The basic version of GWO and the proposed OLBGWO algorithm is discussed in detail in Section 3. To verify the performance of the OLBGWO algorithm, the experiment outcomes are 
analyzed, and a discussion of the previous findings are given in Section 4. Finally, in Section 6, the findings and future directions are stated.

\section{Problem Formulation}

This section of the paper discusses the mathematical modeling of the solar cell and PV module based on SDM and DDM [34,39,52]. Later, the objective function formulation is also discussed to address the parameter identification problem of various models.

\subsection{Solar Photovoltaic Cell Model}

The SDM is typically adopted to study the behavior of the cell, i.e., characteristics of the PV cell. The electrical circuit structure of the single-diode model is illustrated in Fig. 1. The overall current of the photovoltaic cell is presented in Eq. 1.

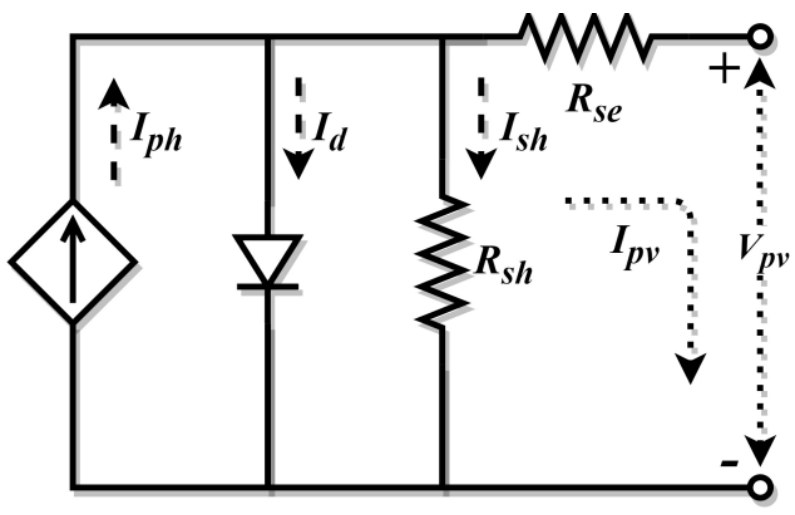

Figure 1. SDM of the solar cell

$I_{p v}=I_{p h}-I_{d}-I_{s h}$

where $I_{p v}$ denotes the total current, $I_{p h}$ represents the photocurrent, $I_{s h}$ represents the current through the shunt resistor, and $I_{d}$ denotes the diode current. Eq. 1 can be rewritten as per Shockley equation as follows.

$I_{p v}=I_{p h}-I_{s d}\left(\frac{q\left(V_{p v}+I_{s e} R_{s e}\right)}{n k T}-1\right)-\frac{V_{p v}+I_{s e} R_{s e}}{R_{s h}}$

where $I_{s d}$ represents the diode's reverse saturation current, $V_{p v}$ denotes the total voltage, $R_{s e}$ and $R_{s h}$ denote the ohmic resistances, $n$ represents the diode ideality factor, $k$ denotes Boltzmann 
constant $\left(1.380653 \times 10^{23} \mathrm{~J} / \mathrm{K}\right), T$ denotes the temperature in $\mathrm{K}$, and $q$ denotes the electron charge $\left(1.60217646 \times 10^{-19} \mathrm{C}\right)$. It can be realized evidently from Eq. 2 that there are five uncertain variables, such as $I_{p h}, I_{s d}, R_{s e}, R_{s h}$, and $n$ for the SDM of the solar cell.

As per the discussion from [39], the SDM of the PV cell does not study the loss due to recombination current effects in the depletion region. Therefore, the precision of the SDM is enhanced by connecting another diode parallel with the existing first diode. The model is called DDM, and it considers the loss due to the recombination. The electrical circuit of such a model is illustrated in Fig. 2. The overall current of the PV cell is presented in Eq. 3.

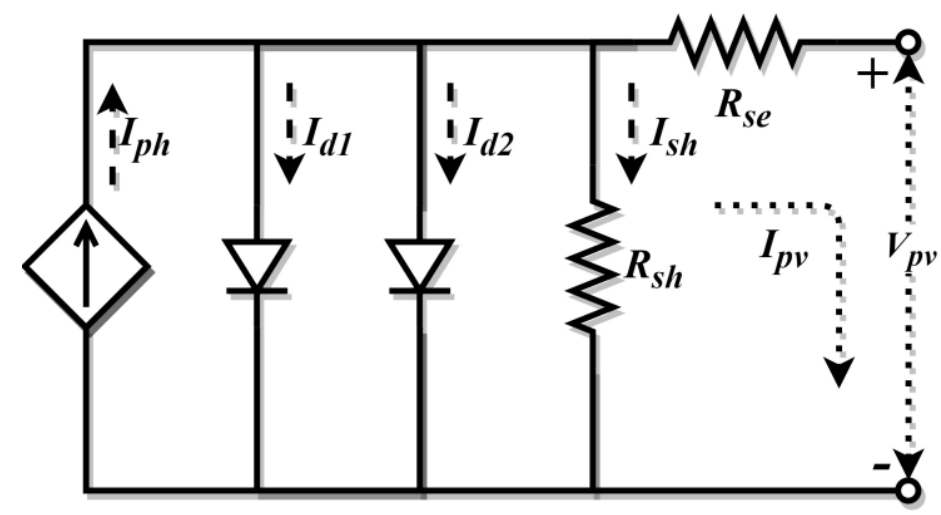

Figure 2. DDM of the solar cell

$I_{p v}=I_{p h}-I_{s d 1}\left(\frac{q\left(V_{p v}+I_{s e} R_{s e}\right)}{n_{1} k T}-1\right)-I_{s d 2}\left(\frac{q\left(V_{p v}+I_{s e} R_{s e}\right)}{n_{2} k T}-1\right)-\frac{V_{p v}+I_{s e} R_{s e}}{R_{s h}}$

where $I_{s d l}$ and $I_{s d 2}$ denote the diode saturation current of diode-1 and diode-2, respectively, $n_{1}$ and $n_{2}$ denote the recombination and diffusion diode ideality factors, respectively. It can be noted evidently from Eq. 3 that there are seven uncertain variables, such as $I_{p h}, I_{s d 1}, I_{s d 2}, R_{s e}, R_{s h}, n_{1}$, and $n_{2}$ for the DDM of the PV cell.

\subsection{Solar Photovoltaic Module Model}

The solar photovoltaic module has been made by connecting $N_{s h}$ parallel-connected cells and $N_{s e}$ number of series-connected cells. The electrical circuit of the photovoltaic panel is illustrated in Fig. 3. The total current of the solar module is presented in Eq. 4. 


$$
I_{p v} / N_{s h}=I_{p h}-I_{s d}\left(\frac{q\left(V_{p v} / N_{s e}+I_{s e} R_{s e} / N_{s h}\right)}{n k T}-1\right)-\frac{V_{p v} / N_{s e}+I_{s e} R_{s e} / N_{s h}}{R_{s h}}
$$



Figure 3. Electrical circuit of photovoltaic model

\subsection{Objective Function Formulation}

The photovoltaic model parameter estimation problem is typically converted into a computational optimization process by optimizing the difference between the estimated value and experimental value. A root-mean-square-error (RMSE) is normally viewed as the error function and is represented in Eq. 5.

$\operatorname{RMSE}(X)=\sqrt{\frac{1}{M} \sum_{i=1}^{M} f\left(V_{p v}, I_{p v}, X\right)^{2}}$

where $X$ denotes the design variables, i.e., five variables for SDM and seven variables for DDM, and $M$ denotes the number of experimental samples.

For SDM of the solar cell, the expression for $f\left(V_{p v}, I_{p v}, X\right)$ is written as follows:

$$
\left.\begin{array}{c}
f\left(V_{p v}, I_{p v}, X\right)=I_{p h}-I_{p v}-I_{s d}\left(\frac{q\left(V_{p v}+I_{s e} R_{s e}\right)}{n k T}-1\right)-\frac{V_{p v}+I_{s e} R_{s e}}{R_{s h}} \\
X=\left\{I_{p h}, I_{s d}, R_{s e}, R_{s h}, n\right\}
\end{array}\right\}
$$


For DDM of the solar cell, the expression for $f\left(V_{p v}, I_{p v}, X\right)$ is written as follows:

$$
\left.\begin{array}{c}
f\left(V_{p v}, I_{p v}, X\right)=I_{p h}-I_{p v}-I_{s d 1}\left(\frac{q\left(V_{p v}+I_{s e} R_{s e}\right)}{n_{1} k T}-1\right)-I_{s d 2}\left(\frac{q\left(V_{p v}+I_{s e} R_{s e}\right)}{n_{2} k T}-1\right)-\frac{V_{p v}+I_{s e} R_{s e}}{R_{s h}} \\
X=\left\{I_{p h}, I_{s d 1}, I_{s d 2}, R_{s e}, R_{s h}, n_{1}, n_{2}\right\}
\end{array}\right\}
$$

For the PV module model, the expression for $f\left(V_{p v}, I_{p v}, X\right)$ is written as follows:

$$
\left.\begin{array}{c}
f\left(V_{p v}, I_{p v}, X\right)=I_{p h}-I_{p v} / N_{s h}-I_{s d}\left(\frac{q\left(V_{p v} / N_{s e}+I_{s e} R_{s e} / N_{s h}\right)}{n k T}-1\right)-\frac{V_{p v} / N_{s e}+I_{s e} R_{s e} / N_{s h}}{R_{s h}} \\
X=\left\{I_{p h}, I_{s d}, R_{s e}, R_{s h}, n\right\}
\end{array}\right\}
$$

\section{Proposed Orthogonal-Learning-Based Gray Wolf Optimizer (OLBGWO) Algorithm}

This section of the paper presents the original version of Gray Wolf Optimizer (GWO) and introduces the orthogonal-learning concept. Then, the integration of OLB and the GWO for solving the parameter estimation problem of various photovoltaic models is discussed.

\subsection{Gray Wolf Optimizer (GWO) Algorithm}

The Gray Wolf Optimizer (GWO) is a recently designed metaheuristic swarm-based algorithm simulating the wolves' swarm's hunting actions [48]. The best individual is named $\alpha$ wolves in the optimizer, the second-and third-best agents are identified respectively as $\beta$ and $\delta$, as well as the further agents, are named $\omega$ wolf. The wolf swarm's activity surrounding the prey is modeled as per Eq. 9 .

$X(l+1)=X_{p}(l)-A \cdot\left|C \cdot X_{p}(l)-X(l)\right|$

where $l$ symbolizes the current iteration, $X$ symbolizes the vector position of the wolf, $X_{p}$ symbolizes the position vector of the prey, $A$ and $C$ are vector coefficients and are written as follows.

$$
\begin{aligned}
& A=2 \cdot a \times r_{1}-a \\
& C=2 \cdot a \times r_{2}
\end{aligned}
$$


$a=2-\frac{2 \times l}{M a x_{-} l}$

where $r_{1}$ and $r_{2}$ are the random values between $[0,1]$ and Max_l denotes the maximum number of iterations. The other individual's positions are updated by the best three wolves, such as $\alpha, \beta$, and $\delta$, and it is mathematically modeled as follows.

$$
\begin{aligned}
& X_{a}=X_{\alpha}-A_{a} \cdot\left|C_{a} \cdot X_{\alpha}-X\right| \\
& X_{b}=X_{\beta}-A_{b} \cdot\left|C_{b} \cdot X_{\beta}-X\right| \\
& X_{c}=X_{\delta}-A_{c} \cdot\left|C_{c} \cdot X_{\delta}-X\right| \\
& X(l+1)=\frac{X_{a}(l)+X_{b}(l)+X_{c}(l)}{3}
\end{aligned}
$$

where $A_{a}, A_{b}$, and $A_{c}$ are similar to $A$ for best three wolves, $C_{a}, C_{b}$, and $C_{c}$ are similar to $C$ for best three wolves, and $X(l+1)$ is the final updated position of the individual.

\subsection{Modified Vector Parameter}

Among all minimization approaches, reaching the global best is a popular and stimulating task [53]. Typically, the optimal route to converge near the least can be separated into two segments. In the initial stages, the populations are spread across the entire search area. In the later stages, the populations take advantage of the value attained to converge on the global best. In GWO, these two phases are balanced by adjusting the parameters $a$ and $A$. The balance between exploitation and exploration is achieved by the parameters $a$ and $A$. Therefore, half of the iteration is allocated to the exploration phase, and the remaining half of the iterations is allocated to the exploitation phase. The local minima problem can be minimized by having large exploration ability. Therefore, in this paper, instead of a linear decrease of the parameter $a$, the exponential decay function is utilized to decrease the vector value $a$. In basic grey wolf optimizer, the value of $a$ linearly decreases from 2 to 0 as given in Eq. 12. In this paper, the value of $a$ is decreased, as presented in Eq. 17. 
$a=2\left(1-\frac{l^{2}}{M a x_{-} l^{2}}\right)$

Based on Eq. 17, the number of iterations utilized for exploitation and exploration is $30 \%$ and $70 \%$, respectively.

\subsection{Orthogonal-Learning-Based (OLB) Approach}

The agent $\alpha$ has a critical part in directing the other followers, and it plays a dynamic part in pushing the entire population in the direction of the global optimum. This paper adopts an OLB approach and builds leadership vectors to lead the agent in the direction of the best. In the OLB mechanism, the position of the individual is updated using an orthogonal-experimental design [54,55]. This paper considers two individuals in the group for the OLB strategy, such as the current evaluation of individual $X_{i}$ and a random individual who is diverse from the current individual. Firstly, the vector solution is separated into $j$ groups; every group is equivalent to a factor, to put on the orthogonal-experimental design strengths to GWO. Secondly, $Q$ levels are created in the position of each dimension to take information in each dimension. Lastly, to produce $M$ solutions, the orthogonal array is used. The procedure is as follows.

Vector Group - Each dimension correlates straight to a factor whenever the vector solution has a limited number of dimensions. When the solution vector's dimensionality is large, reducing the factors needs grouping. A random grouping approach is adopted in this study to better leverage the unique benefits of the population.

Construction Level - For every dimension, $k=1,2, \ldots, \operatorname{dim}, X_{j, k}$, and $X_{i, k}$ are utilized to build the $Q$ levels. It is mathematically modeled as shown in Eq. 18 .

$\operatorname{Level}_{y}=X_{i, k}+\frac{y-1}{Q-1}\left(X_{j, k}-X_{i, k}\right), k=1,2, \ldots, Q$

Generate New Solution - By using the traditionally generated orthogonal array $L_{M}\left(Q^{d}\right)$ and dimensional level construction, new candidate solutions are generated $\left(Z=\left(z_{1}, z_{2}, \ldots, z_{M}\right)\right)$. 
Generate the Best Prediction Solution - By analyzing the fitness, the optimal-level combination experiments are calculated, and it is considered the best prediction solution. Eq. 19 has been used to find the mean fitness for each factor.

$\Delta_{i, j}=\left[\frac{Q}{M} \sum f_{i}\right]$

where $f$ denotes the orthogonal experiment fitness, and compare the fitness at diverse levels and dimensions. Lastly, the low average fitness is selected and is combined to get the best predictive solution. It is possible to generate $M+1$ candidate solutions as per earlier discussions. The smallest fitness is considered as the optimal solution. To avoid the misuse of the OLB approach from interrupting the existing population search space and intervening with the complete search procedure, a trigger constraint is applied to the OLB strategy. For the whole population, a precise experimental approach is to attach a trial matrix. Each cell corresponds to an individual who is utilized to track the stagnation of fitness. The OLBGWO algorithm calculates the objective function when the gray wolf optimizer executes the search agent update procedure and relates the present fitness to one of the earlier iterations. The value of the trial matrix referring to the population is added by 1 if the new fitness is not good. The lack of progress of the $j^{\text {th }}$ individual of the $i^{\text {th }}$ agent is presented in Eq. 20 .

Trial $_{j}^{i}=\left\{\begin{array}{c}\text { Trial }_{j}^{i}+1, \text { if } f i t\left(X_{j, l}^{i}\right) \geq f i t\left(X_{j, l-1}^{i}\right) \\ \text { Trial }_{j}^{i}, \text { if } f i t\left(X_{j, l}^{i}\right)<f i t\left(X_{j, l-1}^{i}\right)\end{array}\right.$

Until executing an OLB procedure on every individual solution, the suggested methodology relates the stagnation limit with the number of stagnations. If the number of stagnations is higher than or equal to the maximum bound, then the operator must execute an OLB update operation, and the maximum bound is assigned to 0. It allows finding the optimal global solution as easy as possible, eliminates undue interference with the search phase, and reduces the difficulty of estimating the algorithm. The Pseudocode of the OLBGWO is given in the Algorithm. In addition, the flowchart of the suggested OLBGWO algorithm is illustrated in Fig. 4. 
Algorithm: Pseudocode of OLBGWO algorithm



End While

Return $X_{\alpha}$

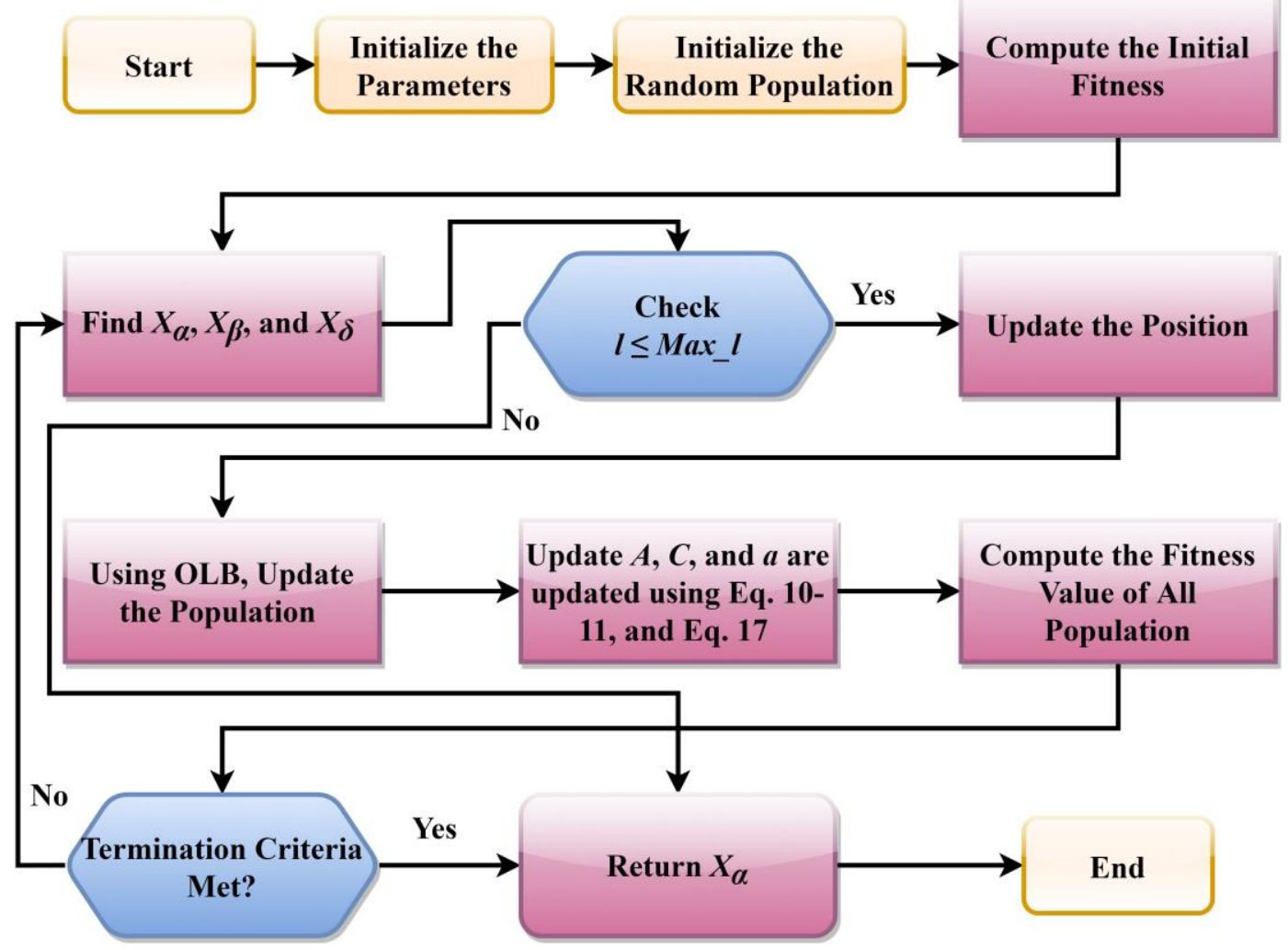

Figure 4. Flowchart of the proposed OLBGWO algorithm 


\section{Simulation Results and Discussions}

In this section, the simulation results are presented to prove the efficiency of the suggested OLBGWO algorithm. For the same, the simulation is done by means of MATLAB R2015a software installed on a PC with a $3.2 \mathrm{GHz}$ clock frequency and 8 GB RAM. The proposed OLBGWO algorithm is directly applied to the parameter identification of various photovoltaic models. The photovoltaic models, such as SDM and DDM of the RTC France Si solar cell (Case1), SDM of the Photowatt-PWP201 PV module (Case-2), SDM of the ST40 PV module (Case3), and SDM of the KC200GT PV module (Case-4) under various operating conditions are considered for verifying the performance of the OLBGWO. The efficiency of the OLBGWO is compared with the other state-of-the-art algorithms, such as PSO, GWO, HHO, SSA, MFO, SMA, and SCA, and the experimental results proved that the OLBGWO gives competitive results and performing better than other stated algorithms. The control parameters of all selected algorithms are listed in Table 1. To have a fair comparison and analysis, each algorithm is run 30 times. The performance comparison of the OLBGWO with other algorithms, in terms of Min, Max, Mean, RMSE, and Wilcoxon signed-rank test (WRT), is discussed in detail. In addition, the upper and lower limits of all design variables for various models are itemized in Table 2.

Table 1. Parameter settings of all selected algorithms

\begin{tabular}{|c|c|c|}
\hline Algorithm & Parameters & Values \\
\hline GWO & $\begin{array}{c}N \\
M a x \_l\end{array}$ & $\begin{array}{c}30 \\
1000\end{array}$ \\
\hline HHO & $\begin{array}{c}N \\
M a x \_l\end{array}$ & $\begin{array}{c}30 \\
1000\end{array}$ \\
\hline SSA & $\begin{array}{c}N \\
M a x \_l\end{array}$ & $\begin{array}{c}30 \\
1000\end{array}$ \\
\hline SCA & $\begin{array}{c}N \\
M a x \_l \\
a\end{array}$ & $\begin{array}{c}30 \\
1000 \\
2\end{array}$ \\
\hline SMA & $\begin{array}{c}N \\
M a x \_l \\
z\end{array}$ & $\begin{array}{c}30 \\
1000 \\
0.03\end{array}$ \\
\hline MFO & $\begin{array}{c}N \\
M a x \_l \\
b\end{array}$ & $\begin{array}{c}30 \\
1000 \\
1\end{array}$ \\
\hline PSO & $\begin{array}{c}N \\
M a x \_l \\
w \\
c_{1} \text { and } c_{2}\end{array}$ & $\begin{array}{c}30 \\
1000 \\
0.5 \\
2\end{array}$ \\
\hline OLBGWO & $\begin{array}{c}N \\
M a x_{-} l \\
a \\
\text { Orthogonal experiment design levels } \\
\text { Orthogonal experiment design factors }\end{array}$ & $\begin{array}{c}30 \\
1000 \\
\text { As per Eq. } 17 \\
3 \\
4\end{array}$ \\
\hline
\end{tabular}


Table 2. Upper and lower limits of all design variables

\begin{tabular}{|c|c|c|c|c|}
\hline \multirow{2}{*}{ Parameters } & \multicolumn{2}{|c|}{ RTC France Si Cell } & \multicolumn{2}{c|}{ Photowatt-PWM201 } \\
\cline { 2 - 5 } & Upper & Lower & Upper & Lower \\
\hline$I_{p h}(A)$ & 1 & 0 & 8 & 0 \\
\hline$R_{s h}(\Omega)$ & 100 & 0 & 1500 & 0 \\
\hline$R_{s e}(\Omega)$ & 0.5 & 0 & 0.4 & 0 \\
\hline$I_{s d}(\mu A)$ & 1 & 0 & 50 & 0 \\
\hline$I_{s d l}, I_{s d 2}(\mu A)$ & 1 & 0 & - & - \\
\hline$n$ & 2 & 1 & 50 & 1 \\
\hline$n_{l}, n_{2}$ & 2 & 1 & - & - \\
\hline
\end{tabular}

\subsection{Case-1: RTC France Si Solar Cell}

In this section, SDM and DDM of the RTC France $\mathrm{Si}$ solar cell are considered for experimentation. The RTC cell is a commercial cell with $57 \mathrm{~mm}$ diameter, and the experimental samples are collected at $1000 \mathrm{~W} / \mathrm{m}^{2}$ irradiation and $33{ }^{\circ} \mathrm{C}$ cell temperature. The upper and lower limits for both models of RTC cell are listed in Table 2. The performance of the suggested OLBGWO algorithm is correlated with PSO, GWO, HHO, SCA, MFO, SSA, and SMA. The above-such algorithms are selected due to the availability of open-source codes, and these algorithms are attracted by the researchers for various applications. Fig. 5 displays the I-V characteristics of the simulation value estimated for both SDM and DDM and experimental value by the proposed OLBGWO algorithm. It can be illustrated that the estimated values obtained by the OLBGWO algorithm closely agree with the experiment samples in the entire voltage sample collection. The relative error (RE) and integral absolute error (IAE) values for the estimated current data and experimental data of both SDM and DDM are illustrated in Fig. 6 and Fig. 7, respectively. The values of RE and IAE are calculated using Eq. 21 and Eq. 22, respectively.

$\mathrm{RE}=\frac{I-I_{S}}{I}$

$\operatorname{IAE}=\left|I-I_{S}\right|$

where $I$ denote the experimental current sample and $I_{s}$ denotes the simulated current value. The calculated IAE and RE values of SDM and DDM are listed in Table 3 and Table 4, respectively. From Table 3-4, it can be observed that the values of IAE are less than 2.50E-03 for SDM and 3.27E-03 for DDM, and RE values are within [-1.20E-03, 1.30E-02] for SDM and [2.03E-02, 1.26E-02] for DDM. From the obtained results, it can be decided that the proposed OLBGWO 
algorithms can correctly replicate the real performance of the SDM and DDM of the PV cell. The sum of the IAE and RE values is $8.27 \mathrm{E}-04$ and $4.77 \mathrm{E}-03$ for SDM, $1.06 \mathrm{E}-03$ and 4.45E-03 for DDM, respectively. It can be seen from Table 3 and Table 4 that the proposed OLBGWO can precisely identify the unknown parameters of the PV cell.

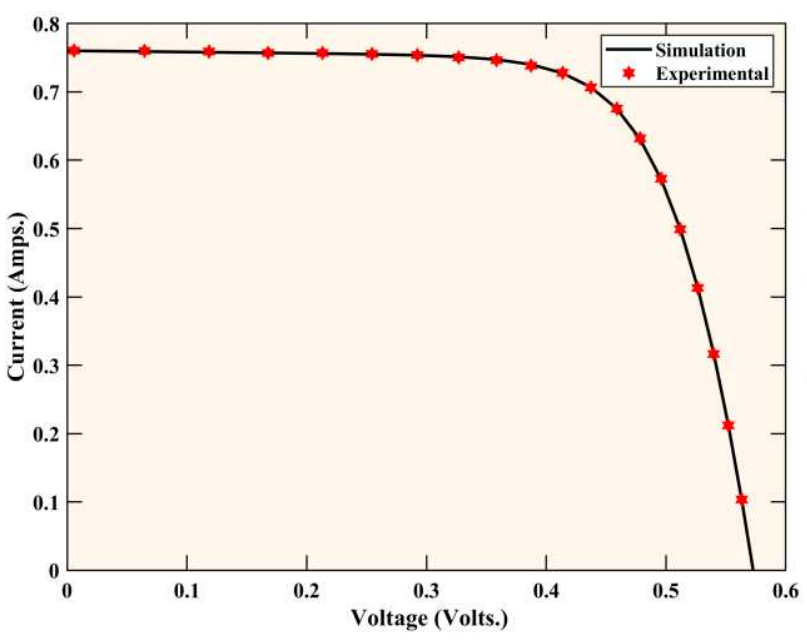

(a)

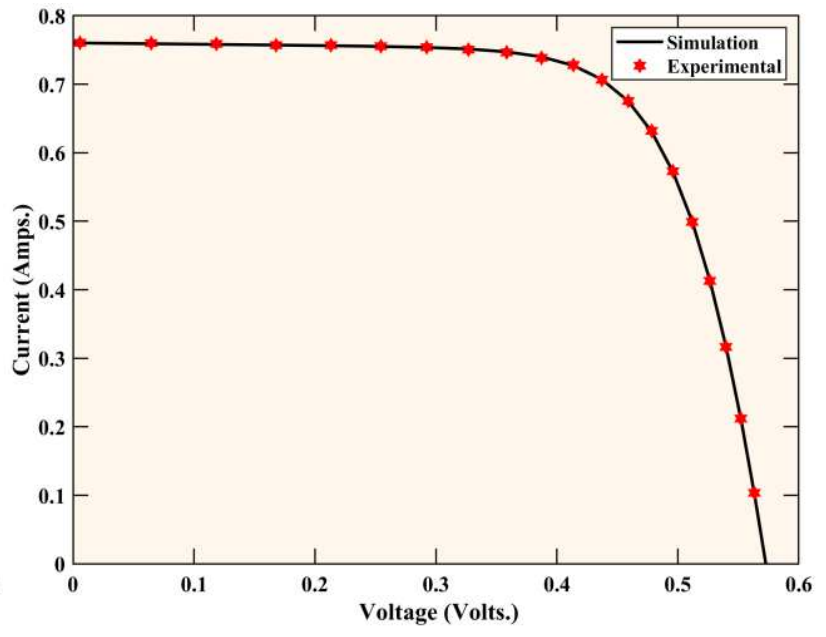

(b)

Figure 5. I-V characteristics of the PV cell; (a) SDM, (b) DDM



(a) 


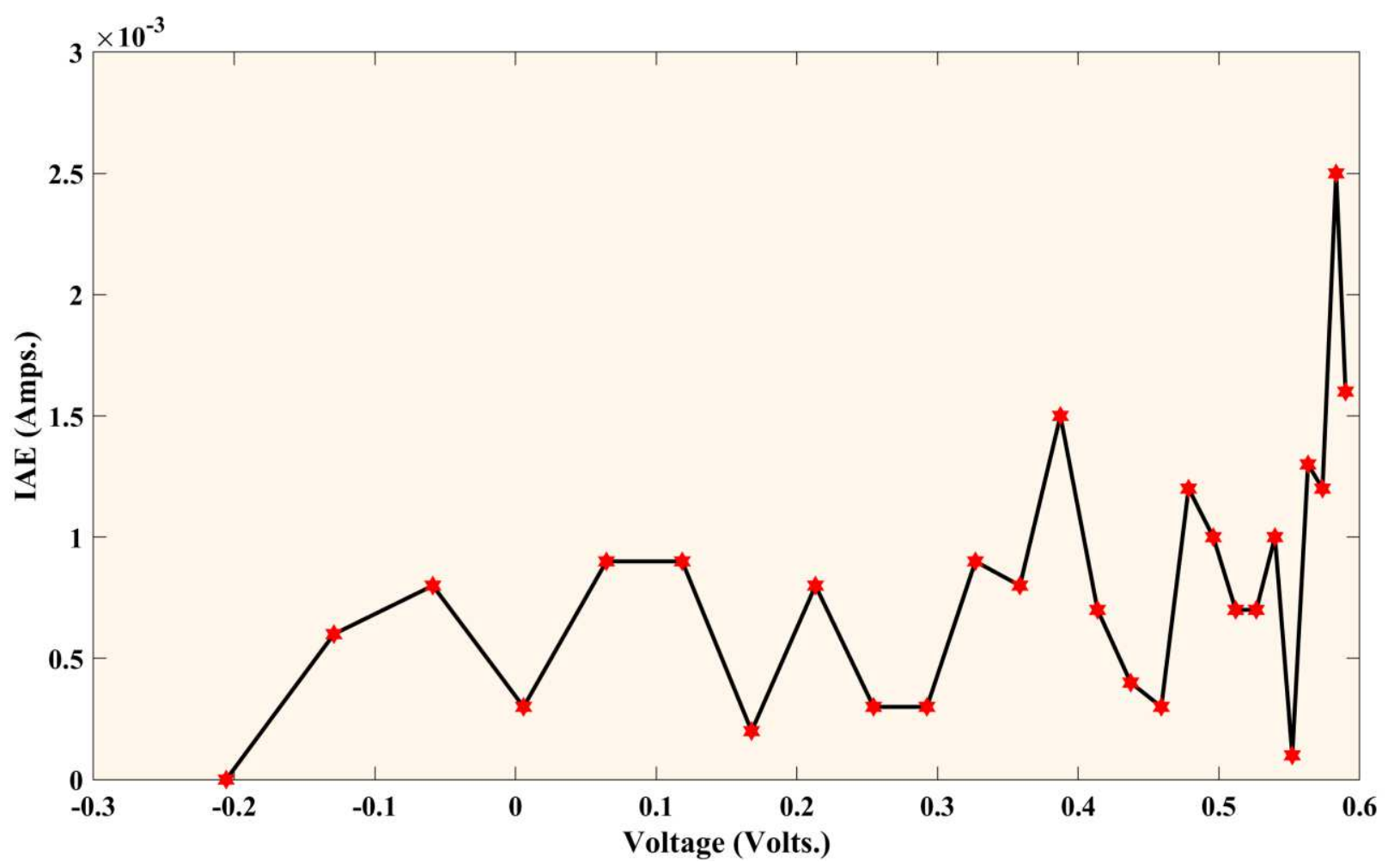

(b)

Figure 6. IAE values of the PV cell; (a) SDM, (b) DDM



(a) 


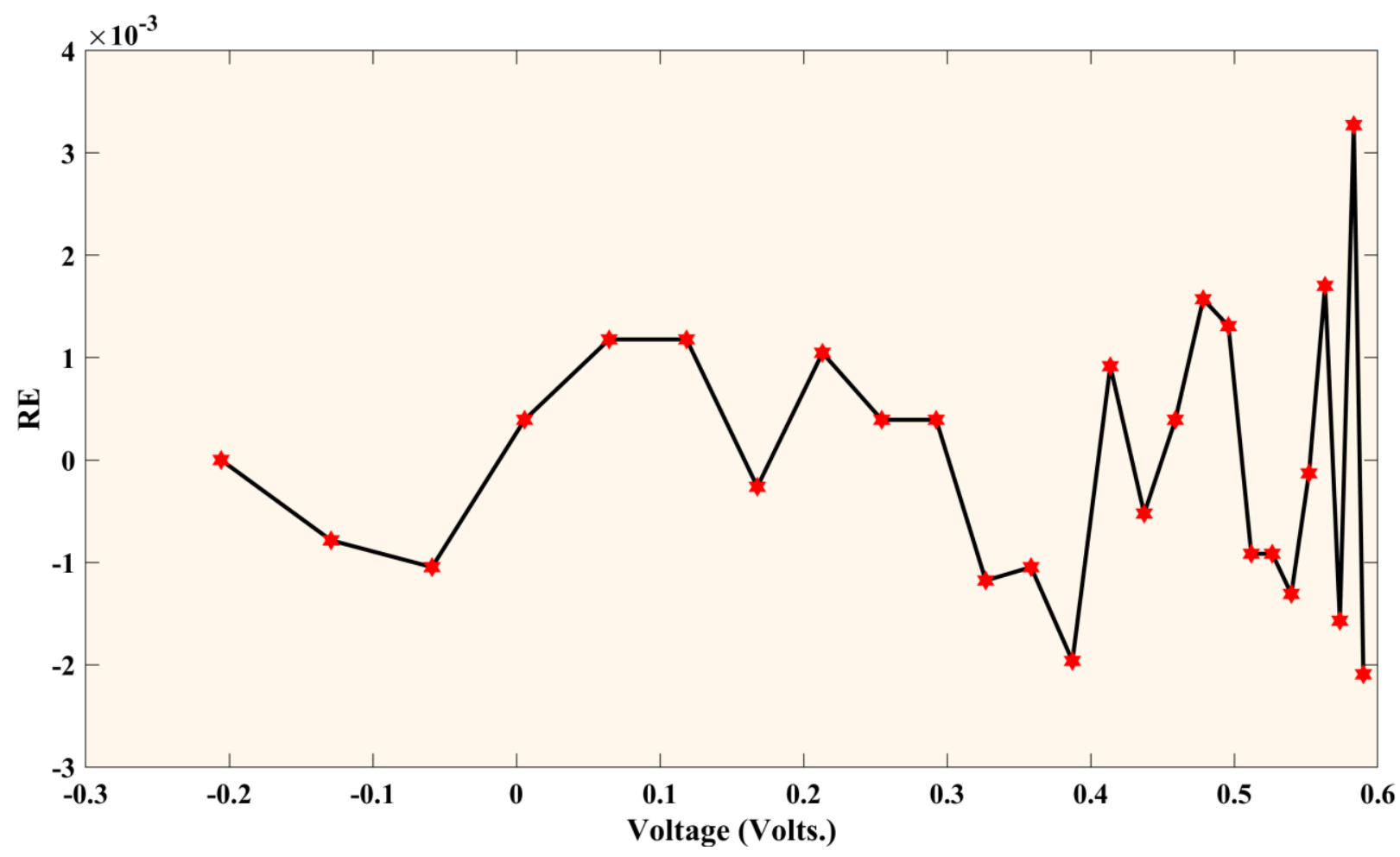

(b)

Figure 7. RE values of the PV cell; (a) SDM, (b) DDM

Table 3. IAE and RE values of the SDM of the PV cell

\begin{tabular}{|c|c|c|c|c|}
\hline $\mathbf{V}(\mathbf{V})$ & $\mathbf{I}(\mathbf{A})$ & $\mathbf{I}_{\mathbf{s}} \mathbf{( A )}$ & $\mathbf{I A E} \mathbf{( A )}$ & $\mathbf{R E}$ \\
\hline-0.2057 & 0.764 & 0.7641 & $1.00 \mathrm{E}-04$ & $-1.31 \mathrm{E}-04$ \\
\hline-0.1291 & 0.762 & 0.7627 & $7.00 \mathrm{E}-04$ & $-9.19 \mathrm{E}-04$ \\
\hline-0.0588 & 0.7605 & 0.7614 & $9.00 \mathrm{E}-04$ & $-1.18 \mathrm{E}-03$ \\
\hline 0.0057 & 0.7605 & 0.7602 & $3.00 \mathrm{E}-04$ & $3.94 \mathrm{E}-04$ \\
\hline 0.0646 & 0.76 & 0.7591 & $9.00 \mathrm{E}-04$ & $1.18 \mathrm{E}-03$ \\
\hline 0.1185 & 0.759 & 0.7580 & $1.00 \mathrm{E}-03$ & $1.32 \mathrm{E}-03$ \\
\hline 0.1678 & 0.757 & 0.7571 & $1.00 \mathrm{E}-04$ & $-1.32 \mathrm{E}-04$ \\
\hline 0.2132 & 0.757 & 0.7561 & $9.00 \mathrm{E}-04$ & $1.19 \mathrm{E}-03$ \\
\hline 0.2545 & 0.7555 & 0.7551 & $4.00 \mathrm{E}-04$ & $5.29 \mathrm{E}-04$ \\
\hline 0.2924 & 0.754 & 0.7537 & $3.00 \mathrm{E}-04$ & $3.98 \mathrm{E}-04$ \\
\hline 0.3269 & 0.7505 & 0.7514 & $9.00 \mathrm{E}-04$ & $-1.20 \mathrm{E}-03$ \\
\hline 0.3585 & 0.7465 & 0.7474 & $9.00 \mathrm{E}-04$ & $-1.21 \mathrm{E}-03$ \\
\hline 0.3873 & 0.7385 & 0.7401 & $1.60 \mathrm{E}-03$ & $-2.17 \mathrm{E}-03$ \\
\hline 0.4137 & 0.728 & 0.7274 & $6.00 \mathrm{E}-04$ & $8.24 \mathrm{E}-04$ \\
\hline 0.4373 & 0.7065 & 0.7070 & $5.00 \mathrm{E}-04$ & $-7.08 \mathrm{E}-04$ \\
\hline
\end{tabular}




\begin{tabular}{|c|c|c|c|c|}
\hline 0.459 & 0.6755 & 0.6753 & $2.00 \mathrm{E}-04$ & $2.96 \mathrm{E}-04$ \\
\hline 0.4784 & 0.632 & 0.6308 & $1.20 \mathrm{E}-03$ & $1.90 \mathrm{E}-03$ \\
\hline 0.496 & 0.573 & 0.5719 & $1.10 \mathrm{E}-03$ & $1.92 \mathrm{E}-03$ \\
\hline 0.5119 & 0.499 & 0.4996 & $6.00 \mathrm{E}-04$ & $-1.20 \mathrm{E}-03$ \\
\hline 0.5265 & 0.413 & 0.4136 & $6.00 \mathrm{E}-04$ & $-1.45 \mathrm{E}-03$ \\
\hline 0.5398 & 0.3165 & 0.3175 & $1.00 \mathrm{E}-03$ & $-3.16 \mathrm{E}-03$ \\
\hline 0.5521 & 0.212 & 0.2122 & $2.00 \mathrm{E}-04$ & $-9.43 \mathrm{E}-04$ \\
\hline 0.5633 & 0.1035 & 0.1023 & $1.20 \mathrm{E}-03$ & $1.16 \mathrm{E}-02$ \\
\hline 0.5736 & -0.01 & -0.0087 & $1.30 \mathrm{E}-03$ & $1.30 \mathrm{E}-02$ \\
\hline 0.5833 & -0.123 & -0.1255 & $2.50 \mathrm{E}-03$ & $-2.03 \mathrm{E}-02$ \\
\hline 0.59 & -0.21 & -0.2085 & $1.50 \mathrm{E}-03$ & $7.14 \mathrm{E}-03$ \\
\hline & Sum & $8.27 \mathrm{E}-04$ & $4.77 \mathrm{E}-03$ \\
\hline
\end{tabular}

Table 4. IAE and RE values of the DDM of the PV cell

\begin{tabular}{|c|c|c|c|c|}
\hline $\mathrm{V}(\mathrm{V})$ & I (A) & $I_{s}(A)$ & IAE (A) & RE \\
\hline-0.2057 & 0.764 & 0.7640 & $0.00 \mathrm{E}+00$ & $0.00 \mathrm{E}+00$ \\
\hline-0.1291 & 0.762 & 0.7626 & 7.85E-04 & $-7.87 \mathrm{E}-04$ \\
\hline-0.0588 & 0.7605 & 0.7613 & $1.05 \mathrm{E}-03$ & $-1.05 \mathrm{E}-03$ \\
\hline 0.0057 & 0.7605 & 0.7602 & $3.93 \mathrm{E}-04$ & $3.94 \mathrm{E}-04$ \\
\hline 0.0646 & 0.76 & 0.7591 & 1.18E-03 & $1.18 \mathrm{E}-03$ \\
\hline 0.1185 & 0.759 & 0.7581 & $1.18 \mathrm{E}-03$ & 1.19E-03 \\
\hline 0.1678 & 0.757 & 0.7572 & $2.62 \mathrm{E}-04$ & $-2.64 \mathrm{E}-04$ \\
\hline 0.2132 & 0.757 & 0.7562 & $1.05 \mathrm{E}-03$ & $1.06 \mathrm{E}-03$ \\
\hline 0.2545 & 0.7555 & 0.7552 & 3.93E-04 & $3.97 \mathrm{E}-04$ \\
\hline 0.2924 & 0.754 & 0.7537 & 3.93E-04 & 3.98E-04 \\
\hline 0.3269 & 0.7505 & 0.7514 & $1.18 \mathrm{E}-03$ & $-1.20 \mathrm{E}-03$ \\
\hline 0.3585 & 0.7465 & 0.7473 & $1.05 \mathrm{E}-03$ & $-1.07 \mathrm{E}-03$ \\
\hline 0.3873 & 0.7385 & 0.7400 & $1.96 \mathrm{E}-03$ & $-2.03 \mathrm{E}-03$ \\
\hline 0.4137 & 0.728 & 0.7273 & $9.16 \mathrm{E}-04$ & $9.62 \mathrm{E}-04$ \\
\hline 0.4373 & 0.7065 & 0.7069 & 5.24E-04 & $-5.66 \mathrm{E}-04$ \\
\hline 0.459 & 0.6755 & 0.6752 & 3.93E-04 & 4.44E-04 \\
\hline 0.4784 & 0.632 & 0.6308 & $1.57 \mathrm{E}-03$ & $1.90 \mathrm{E}-03$ \\
\hline 0.496 & 0.573 & 0.5720 & $1.31 \mathrm{E}-03$ & $1.75 \mathrm{E}-03$ \\
\hline 0.5119 & 0.499 & 0.4997 & $9.16 \mathrm{E}-04$ & $-1.40 \mathrm{E}-03$ \\
\hline 0.5265 & 0.413 & 0.4137 & $9.16 \mathrm{E}-04$ & $-1.69 \mathrm{E}-03$ \\
\hline 0.5398 & 0.3165 & 0.3175 & $1.31 \mathrm{E}-03$ & $-3.16 \mathrm{E}-03$ \\
\hline 0.5521 & 0.212 & 0.2121 & $1.31 \mathrm{E}-04$ & $-4.72 E-04$ \\
\hline 0.5633 & 0.1035 & 0.1022 & $1.70 \mathrm{E}-03$ & $1.26 \mathrm{E}-02$ \\
\hline 0.5736 & -0.01 & -0.0088 & $1.57 \mathrm{E}-03$ & $1.20 \mathrm{E}-02$ \\
\hline 0.5833 & -0.123 & -0.1255 & $3.27 \mathrm{E}-03$ & $-2.03 \mathrm{E}-02$ \\
\hline 0.59 & -0.21 & -0.2084 & 2.09E-03 & $7.62 \mathrm{E}-03$ \\
\hline \multicolumn{3}{|c|}{ Sum } & $1.06 \mathrm{E}-03$ & $4.45 \mathrm{E}-03$ \\
\hline
\end{tabular}


The obtained RMSE values by the suggested OLBGWO algorithm and other selected algorithms are listed in Table 5 and Table 6 after 30 individual runs of all algorithms. The statistical analysis of RMSE values of SDM and DDM, including Max, Min, Mean, standard deviation (SD), and runtime (RT) of all algorithms are listed in Table 5 and Table 6, respectively. From Table 5-6, it is observed that the OLBGWO displays better performance as per Min, Max, SD, and RT values. Therefore, it is concluded that the proposed OLBGWL algorithm can display more stable and reliable results compared to other competitive algorithms. The convergence of both SDM and DDM of the PV cell is illustrated in Fig. 8. From Fig. 8, it can be seen that the convergence speed of the proposed OLBGWO algorithm is faster than the other selected algorithms. Few algorithms, such as PSO, SSA, GWO, and SCA, are trapped into local optima too early. Therefore, as per the convergence speed and the solution accuracy, the suggested OLBGWO technique performs better than other competitors.

Table 5. Statistical values for the SDM of the PV cell

\begin{tabular}{|c|c|c|c|c|c|c|}
\hline Algorithm & Min & Max & Mean & SD & RT & Remarks \\
\hline OLBGWO & 0.000986 & 0.000986 & 0.000986 & $1.4 \mathrm{E}-08$ & 17.28646 & \\
\hline SMA & 0.000986 & 0.001031 & 0.001001 & $2.62 \mathrm{E}-05$ & 22.88021 & + \\
\hline GWO & 0.001005 & 0.003252 & 0.00201 & 0.001142 & 17.25 & + \\
\hline PSO & 0.002296 & 0.00658 & 0.004269 & 0.002161 & 43.25521 & + \\
\hline SCA & 0.011492 & 0.042765 & 0.031969 & 0.017742 & 56.53125 & + \\
\hline SSA & 0.003426 & 0.041475 & 0.019636 & 0.01964 & 45.59896 & + \\
\hline HHO & 0.000989 & 0.000991 & 0.00099 & $1.16 \mathrm{E}-06$ & 60.35938 & + \\
\hline MFO & 0.001125 & 0.001707 & 0.001476 & 0.000309 & 17.86979 & + \\
\hline
\end{tabular}

Table 6. Statistical values for the DDM of the PV cell

\begin{tabular}{|c|c|c|c|c|c|c|}
\hline Algorithm & Min & Max & Mean & SD & RT & Remarks \\
\hline OLBGWO & 0.000983 & 0.000986 & 0.000985 & $1.78 \mathrm{E}-06$ & 17.48438 & \\
\hline SMA & 0.000984 & 0.000986 & 0.000985 & $9.01 \mathrm{E}-06$ & 22.76563 & + \\
\hline GWO & 0.00321 & 0.007358 & 0.005242 & 0.002075 & 17.33333 & + \\
\hline PSO & 0.002093 & 0.013568 & 0.006233 & 0.00637 & 43.55208 & + \\
\hline SCA & 0.040949 & 0.046102 & 0.04334 & 0.002596 & 57.04167 & + \\
\hline SSA & 0.001365 & 0.004889 & 0.003331 & 0.001797 & 45.34375 & + \\
\hline HHO & 0.000986 & 0.001006 & 0.000993 & $1.13 \mathrm{E}-05$ & 60.94271 & + \\
\hline MFO & 0.001018 & 0.001941 & 0.001447 & 0.000465 & 18.14063 & + \\
\hline
\end{tabular}




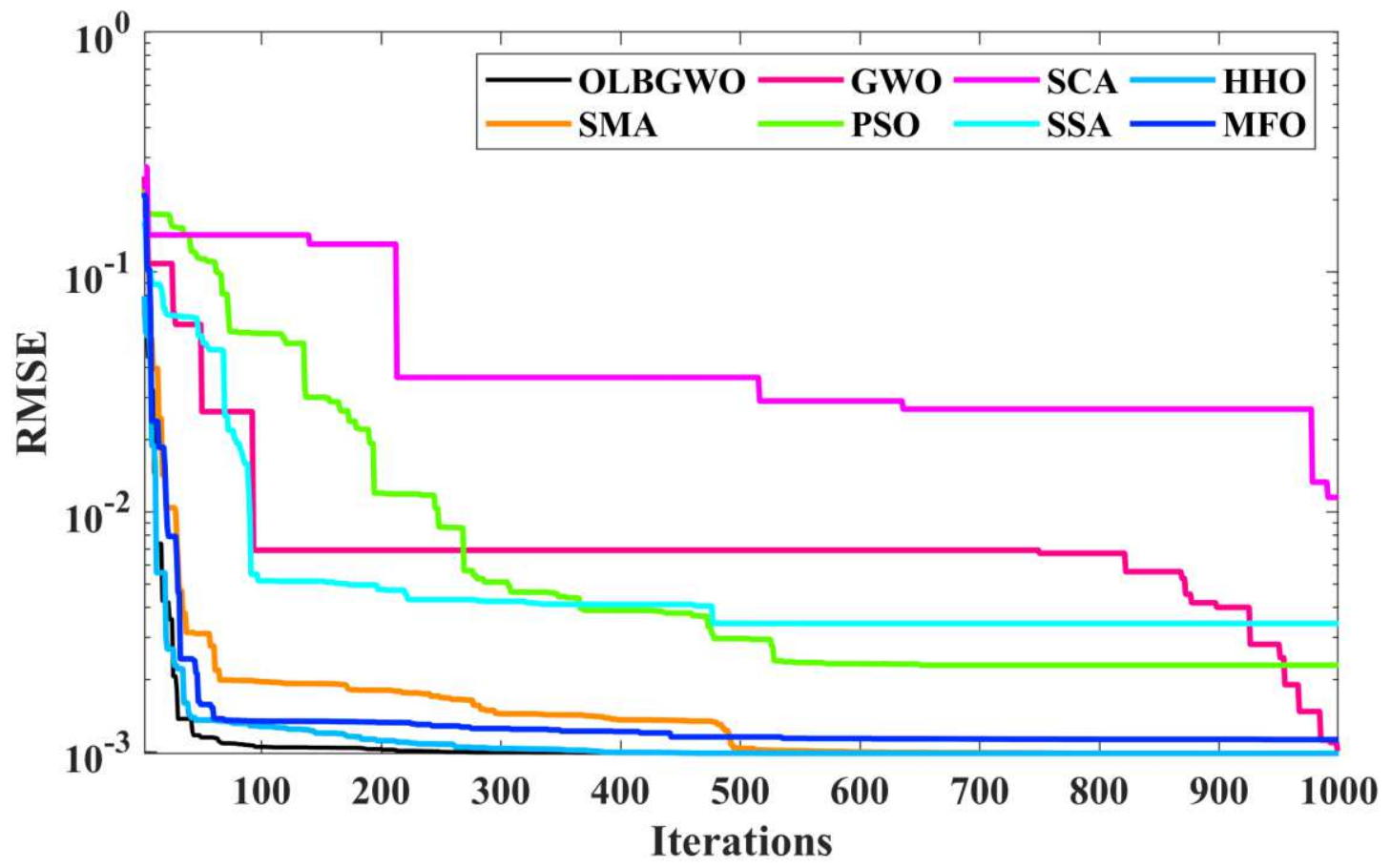

(a)

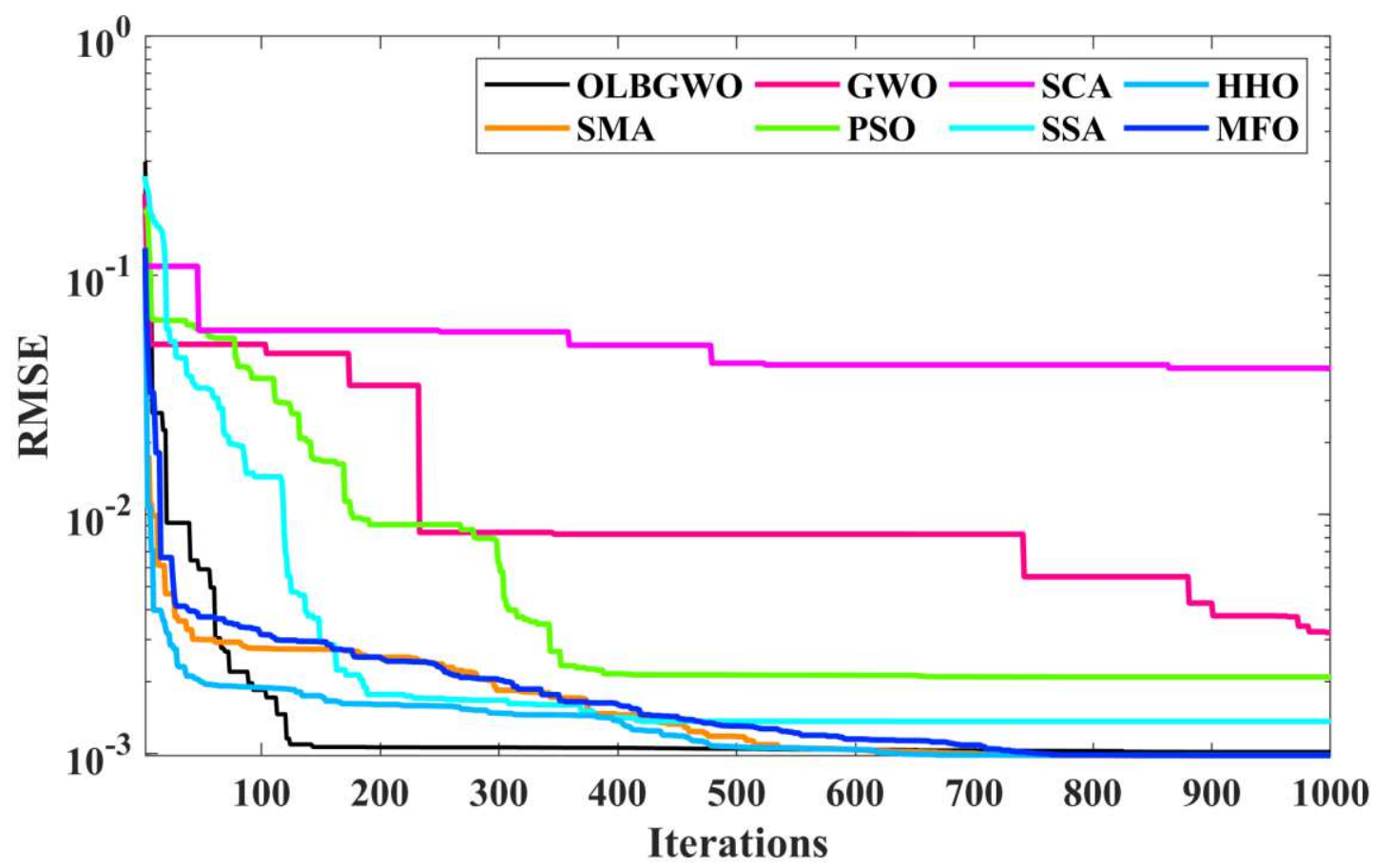

(b)

Figure 8. Convergence curve of all algorithms; (a) SDM, (b) DDM

The best estimated variables and RMSE acquired from all algorithms, including OLBGWO for SDM and DDM, are listed in Table 7 and Table 8, respectively. From Table 7-8, it can be 
observed that the proposed OLBGWO algorithm finds the best RMSE value, i.e., 9.8602E-04 for SDM and 9.8254E-04 for DDM, which are less than the other selected algorithms. Therefore, it can be concluded that the suggested OLBGWO algorithm is a robust tool to identify the unknown parameters of the PV cell.

Table 7. Optimal parameters found by selected algorithms for the SDM of the PV cell

\begin{tabular}{|c|c|c|c|c|c|c|c|}
\hline Algorithm & $\boldsymbol{I}_{\boldsymbol{p}}(\mathbf{A})$ & $\boldsymbol{a}$ & $\boldsymbol{R}_{\boldsymbol{s} \boldsymbol{}}(\mathbf{\Omega})$ & $\boldsymbol{R}_{\boldsymbol{s} \boldsymbol{}}(\mathbf{\Omega})$ & $\boldsymbol{I}_{\boldsymbol{s} \boldsymbol{d}}(\boldsymbol{\mu A} \mathbf{A})$ & $\boldsymbol{R M S E}$ & sig \\
\hline OLBGWO & 0.760775446 & 1.481184371 & 53.71884978 & 0.036377049 & $3.23023 \mathrm{E}-07$ & 0.000986022 & \\
\hline SMA & 0.76077549 & 1.481182374 & 53.7190549 & 0.036377158 & $3.23017 \mathrm{E}-07$ & 0.000986029 & + \\
\hline GWO & 0.760594421 & 1.488054233 & 55.38693081 & 0.036041973 & $3.45601 \mathrm{E}-07$ & 0.001005009 & + \\
\hline PSO & 0.760651309 & 1.589218609 & 88.03038933 & 0.031629759 & $8.76874 \mathrm{E}-07$ & 0.002296352 & + \\
\hline SCA & 0.746825193 & 1.508266992 & 65.48976491 & 0.034759925 & $4.15848 \mathrm{E}-07$ & 0.011492196 & + \\
\hline SSA & 0.762156665 & 1.57813106 & 85.30097679 & 0.03408091 & $7.98838 \mathrm{E}-07$ & 0.00342559 & + \\
\hline HHO & 0.760775453 & 1.481186339 & 53.71962251 & 0.036376993 & $3.2303 \mathrm{E}-07$ & 0.00098904 & + \\
\hline MFO & 0.760273931 & 1.504819789 & 69.52501857 & 0.035540698 & $4.07479 \mathrm{E}-07$ & 0.001124854 & + \\
\hline
\end{tabular}

Table 8. Optimal parameters found by selected algorithms for the DDM of the PV cell

\begin{tabular}{|c|c|c|c|c|c|c|c|c|c|}
\hline Algorithm & $\boldsymbol{I}_{p}(\mathbf{A})$ & $a_{1}$ & $a_{2}$ & $R_{s h}(\Omega)$ & $\boldsymbol{R}_{s e}(\boldsymbol{\Omega})$ & $I_{s d I}(\mu \mathrm{A})$ & $I_{s d 2}(\boldsymbol{\mu} \mathbf{A})$ & RSME & sig \\
\hline OLBGWO & 0.760781114 & 1.451328017 & 1.961750012 & 55.30775593 & 0.036722397 & $2.25939 \mathrm{E}-07$ & $6.43151 \mathrm{E}-07$ & 0.000982556 & \\
\hline GWO & 0.765070224 & 1.999417133 & 1.491312557 & 29.33874544 & 0.034524345 & $6.7826 \mathrm{E}-07$ & 3.36824E-07 & 0.003209823 & + \\
\hline SCA & 0.743316562 & 1.621502389 & 1 & 11.55798222 & 0.014902051 & 0.000001 & $9.51767 \mathrm{E}-08$ & 0.04094904 & + \\
\hline SSA & 0.759613138 & 1.658890834 & 1.498387292 & 92.4022982 & 0.035572451 & $1.0924 \mathrm{E}-07$ & 3.56849E-07 & 0.001364675 & + \\
\hline $\mathrm{HHO}$ & 0.760777324 & 1.999999993 & 1.481062192 & 53.67960509 & 0.036380366 & $1.07041 \mathrm{E}-09$ & $3.22607 \mathrm{E}-07$ & 0.000986014 & + \\
\hline
\end{tabular}

\subsection{Case-2: Photowatt PWP-201 PV Module}

In this section, the SDM of the Photowatt PWP-201 PV model is deliberated for experimentation. The Photowatt PWP-201 is a commercial PV module with 36 polycrystalline PV cells in series, and the experimental samples are collected at $1000 \mathrm{~W} / \mathrm{m}^{2}$ irradiation and 45 ${ }^{\circ} \mathrm{C}$ cell temperature. The upper and lower limits for both models are listed in Table 2. The 
performance of the proposed OLBGWO algorithm is compared with PSO, GWO, HHO, SCA, MFO, SSA, and SMA. Fig. 9 displays the I-V characteristics of the simulation value estimated for the SDM and experimental value by the proposed OLBGWO algorithm. It can be illustrated that the estimated values obtained by the OLBGWO algorithm closely agree with the experiment samples in the entire voltage sample collection. The RE and IAE values for the estimated current value and experimental values of SDM is illustrated in Fig. 10 and Fig. 11, respectively. The calculated IAE and RE values of SDM are listed in Table 9. From Table 9, it can be seen that the values of IAE are less than 4.80E-03, and the values of RE are within the range of [-1.65E-03, 4.65E-03]. From these results, it can be decided that the suggested OLBGWO algorithms can correctly replicate the real performance of the SDM of the PV module. The sum of the IAE and $\mathrm{RE}$ values is $1.97 \mathrm{E}-03$ and $1.44 \mathrm{E}-03$, respectively. It can be observed from Table 9 that the suggested OLBGWO can precisely estimate the unknown parameters of the PV cell.

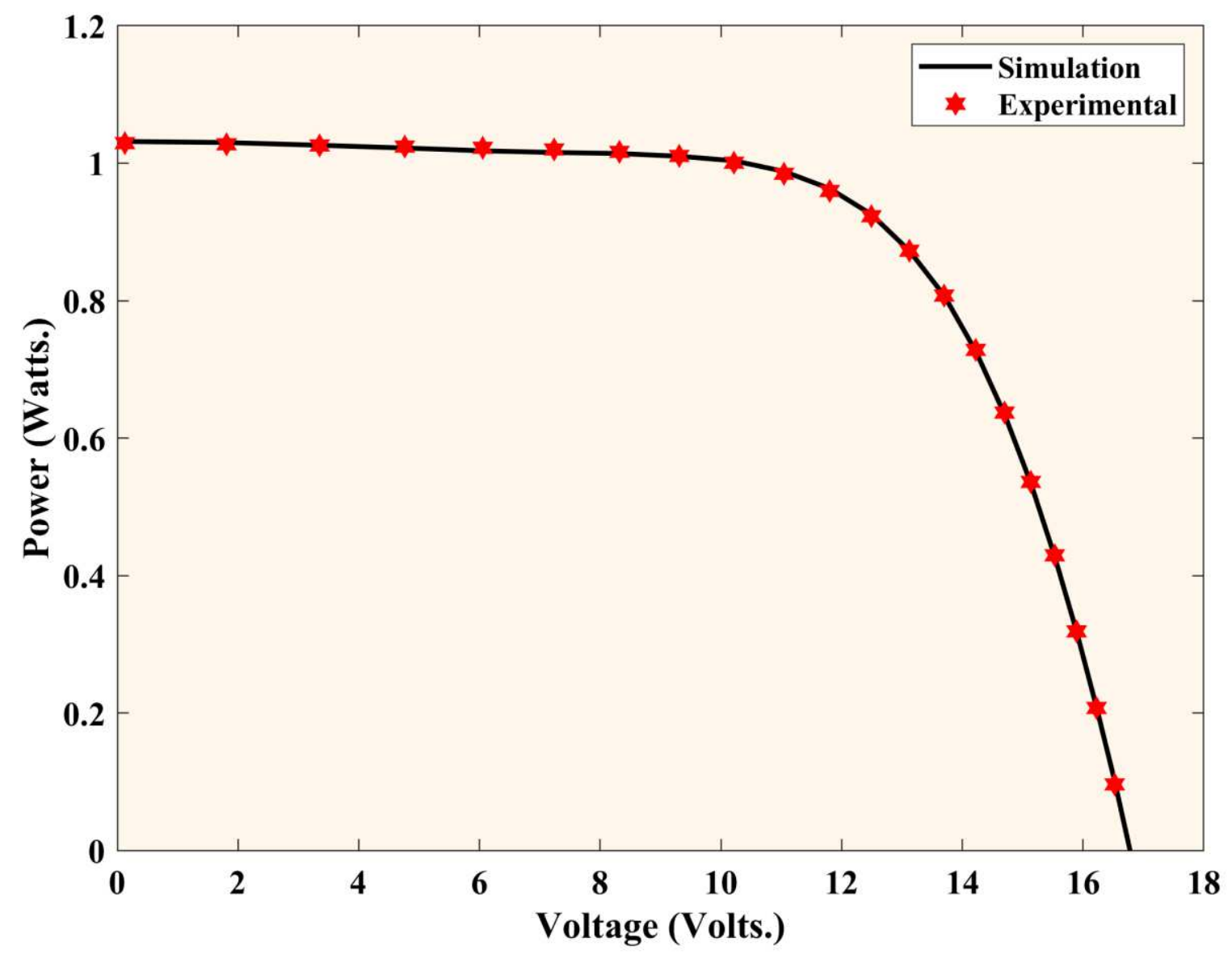

Figure 9. I-V characteristics of the PV module model 


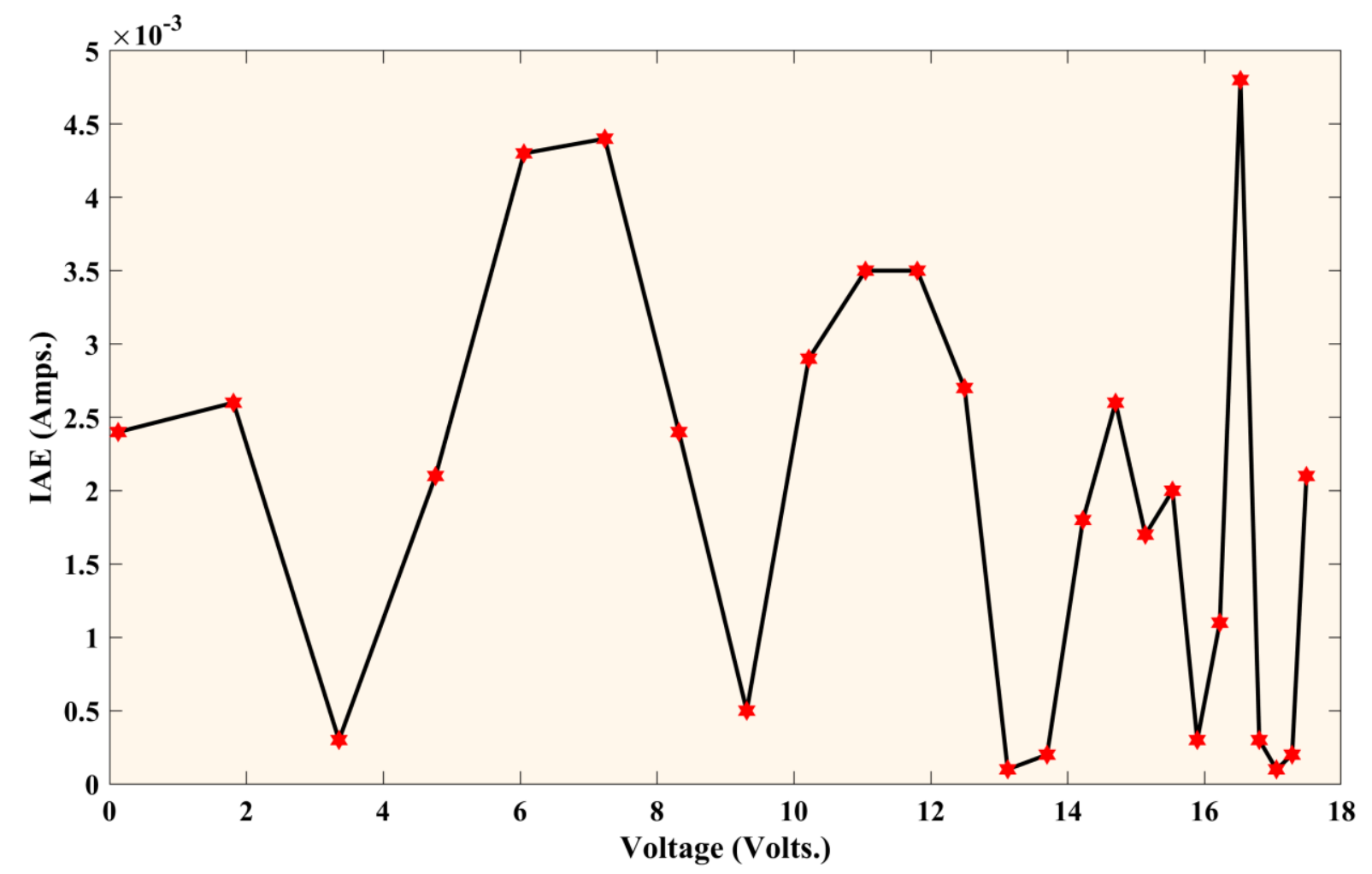

Figure 10. IAE values of the PV module model



Figure 11. RE values of the PV module model 
Table 9. IAE and RE values of the PV module model

\begin{tabular}{|c|c|c|c|c|}
\hline $\mathbf{V}(\mathbf{V})$ & $\mathbf{I}(\mathbf{A})$ & $\mathbf{I}_{\mathbf{s}}(\mathbf{A})$ & $\mathbf{I A E}(\mathbf{A})$ & $\mathbf{R E}$ \\
\hline 0.1248 & 1.0315 & 1.0291 & $2.40 \mathrm{E}-03$ & $2.33 \mathrm{E}-03$ \\
\hline 1.8093 & 1.0300 & 1.0274 & $2.60 \mathrm{E}-03$ & $2.52 \mathrm{E}-03$ \\
\hline 3.3511 & 1.0260 & 1.0257 & $3.00 \mathrm{E}-04$ & $2.91 \mathrm{E}-04$ \\
\hline 4.7622 & 1.0220 & 1.0241 & $2.10 \mathrm{E}-03$ & $2.04 \mathrm{E}-03$ \\
\hline 6.0538 & 1.0180 & 1.0223 & $4.30 \mathrm{E}-03$ & $4.17 \mathrm{E}-03$ \\
\hline 7.2364 & 1.0155 & 1.0199 & $4.40 \mathrm{E}-03$ & $4.27 \mathrm{E}-03$ \\
\hline 8.3189 & 1.0140 & 1.0164 & $2.40 \mathrm{E}-03$ & $2.33 \mathrm{E}-03$ \\
\hline 9.3097 & 1.0100 & 1.0105 & $5.00 \mathrm{E}-04$ & $4.85 \mathrm{E}-04$ \\
\hline 10.2163 & 1.0035 & 1.0006 & $2.90 \mathrm{E}-03$ & $2.81 \mathrm{E}-03$ \\
\hline 11.0449 & 0.9880 & 0.9845 & $3.50 \mathrm{E}-03$ & $3.39 \mathrm{E}-03$ \\
\hline 11.8018 & 0.9630 & 0.9595 & $3.50 \mathrm{E}-03$ & $3.39 \mathrm{E}-03$ \\
\hline 12.4929 & 0.9255 & 0.9228 & $2.70 \mathrm{E}-03$ & $2.62 \mathrm{E}-03$ \\
\hline 13.1231 & 0.8725 & 0.8726 & $1.00 \mathrm{E}-04$ & $-9.69 \mathrm{E}-05$ \\
\hline 13.6983 & 0.8075 & 0.8073 & $2.00 \mathrm{E}-04$ & $1.94 \mathrm{E}-04$ \\
\hline 14.2221 & 0.7265 & 0.7283 & $1.80 \mathrm{E}-03$ & $1.75 \mathrm{E}-03$ \\
\hline 14.6995 & 0.6345 & 0.6371 & $2.60 \mathrm{E}-03$ & $2.52 \mathrm{E}-03$ \\
\hline 15.1346 & 0.5345 & 0.5362 & $1.70 \mathrm{E}-03$ & $-1.65 \mathrm{E}-03$ \\
\hline 15.5311 & 0.4275 & 0.4295 & $2.00 \mathrm{E}-03$ & $-1.94 \mathrm{E}-03$ \\
\hline 15.8929 & 0.3185 & 0.3188 & $3.00 \mathrm{E}-04$ & $-2.91 \mathrm{E}-04$ \\
\hline 16.2229 & 0.2085 & 0.2074 & $1.10 \mathrm{E}-03$ & $1.07 \mathrm{E}-03$ \\
\hline 16.5241 & 0.1010 & 0.0962 & $4.80 \mathrm{E}-03$ & $4.65 \mathrm{E}-03$ \\
\hline 16.7987 & -0.0080 & -0.0083 & $3.00 \mathrm{E}-04$ & $2.91 \mathrm{E}-04$ \\
\hline 17.0499 & -0.1110 & -0.1109 & $1.00 \mathrm{E}-04$ & $-9.69 \mathrm{E}-05$ \\
\hline 17.2793 & -0.2090 & -0.2092 & $2.00 \mathrm{E}-04$ & $1.94 \mathrm{E}-04$ \\
\hline 17.4885 & -0.3030 & -0.3009 & $2.10 \mathrm{E}-03$ & $-2.04 \mathrm{E}-03$ \\
\hline 0.1248 & 1.0315 & 1.0291 & $2.40 \mathrm{E}-03$ & $2.33 \mathrm{E}-03$ \\
\hline & Sum & & $1.97 \mathrm{E}-03$ & $1.44 \mathrm{E}-03$ \\
\hline
\end{tabular}

The obtained RMSE values by the proposed OLBGWO algorithm and other selected algorithms are listed in Table 10 after 30 individual runs of all algorithms. The statistical analysis of RMSE values of the PV module model, including Min, Max, Mean, Median, SD, and RT of all algorithms are listed in Table 10. Table 10 shows that the OLBGWO displays better performance as per Min, Max, SD, and RT values. Therefore, it is concluded that the proposed OLBGWL algorithm can display more stable and reliable results compared to other competitive algorithms. The convergence of the PV module model is illustrated in Fig. 12. From Fig. 12, it can be noticed that the convergence rate of the suggested OLBGWO algorithm is faster than the other selected algorithms. Few algorithms, such as PSO, SCA, and GWO, are trapped into local 
optima too early. Therefore, as per the convergence rate and the solution accuracy, the suggested OLBGWO algorithm performs better than other competitors.

Table 10. Statistical values for the PV module model

\begin{tabular}{|c|c|c|c|c|c|c|}
\hline Algorithm & Min & Max & Mean & SD & RT & Remarks \\
\hline OLBGWO & 0.0024 & 0.0024 & 0.0024 & $2.4284 \mathrm{e}-09$ & 14.6667 & \\
\hline SMA & 0.0025 & 0.0028 & 0.0027 & $5.4900 \mathrm{e}-06$ & 20.0208 & + \\
\hline GWO & 0.0027 & 0.0090 & 0.0055 & 0.0032 & 14.5156 & + \\
\hline PSO & 0.0717 & 0.2799 & 0.2086 & 0.1187 & 37.6927 & + \\
\hline SCA & 0.0133 & 0.2743 & 0.1210 & 0.1363 & 48.6563 & + \\
\hline SSA & 0.0026 & 0.0771 & 0.0294 & 0.0414 & 40.5677 & + \\
\hline HHO & 0.0026 & 0.0029 & 0.0026 & $5.8604 \mathrm{e}-06$ & 52.1510 & + \\
\hline MFO & 0.0025 & 0.2743 & 0.0935 & 0.1566 & 15.3177 & + \\
\hline
\end{tabular}



Figure 12. Convergence curve of all algorithms for the PV module model

The optimal identified variables and RMSE obtained from all algorithms, including OLBGWO for the photovoltaic panel model, are listed in Table 11. Table 11 shows that the proposed OLBGWO algorithm obtains the best RMSE, i.e., 1.256E-03, which are less than the other selected algorithms. Therefore, it can be concluded that the suggested OLBGWO technique is a robust tool to identify the unknown parameters of the PV module. 
Table 11. Optimal parameters obtained by all algorithms for the PV module model

\begin{tabular}{|c|c|c|c|c|c|c|c|}
\hline Algorithm & $\boldsymbol{I}_{\boldsymbol{p}}(\mathbf{A})$ & $\boldsymbol{a}$ & $\boldsymbol{R}_{\boldsymbol{s} \boldsymbol{h}}(\boldsymbol{\Omega})$ & $\boldsymbol{R}_{\boldsymbol{s} \boldsymbol{e}}(\boldsymbol{\Omega})$ & $\boldsymbol{I}_{\boldsymbol{s} \boldsymbol{d}}(\boldsymbol{\mu A})$ & $\boldsymbol{R M S E}$ & $\boldsymbol{s i g}$ \\
\hline OLBGWO & 1.0305 & 48.6428 & 981.9818 & 1.2013 & $3.4823 \mathrm{e}-06$ & 0.0024 & \\
\hline SMA & 1.0305 & 48.6855 & 988.9833 & 1.2013 & $3.497 \mathrm{e}-06$ & 0.0025 & + \\
\hline GWO & 1.0305 & 50 & $1.1875 \mathrm{e}+03$ & 1.1613 & $4.9087 \mathrm{e}-06$ & 0.0027 & + \\
\hline PSO & 0.9792 & 50 & 2000 & 0.3858 & $4.5303 \mathrm{e}-06$ & 0.0717 & + \\
\hline SCA & 1.0216 & 50 & 989.3061 & 1.1157 & $4.7498 \mathrm{e}-06$ & 0.0133 & + \\
\hline SSA & 1.0278 & 49.8818 & $1.8271 \mathrm{e}+03$ & 1.1700 & $4.7807 \mathrm{e}-06$ & 0.0026 & + \\
\hline HHO & 1.0305 & 48.6978 & 987.4788 & 1.2014 & $3.5796 \mathrm{e}-06$ & 0.0026 & + \\
\hline MFO & 1.0289 & 49.5598 & $1.3652 \mathrm{e}+03$ & 1.1754 & $4.4060 \mathrm{e}-06$ & 0.0025 & + \\
\hline
\end{tabular}

\subsection{Case-3: Commercial ST40 PV Module}

The OLBGWO is also examined for optimizing the problems of parameter identification of the commercial ST40 PV module. The experimental values of the ST40 commercial module under different temperature and irradiance conditions are collected to confirm the efficiency of the proposed OLBGWO algorithm. The ST40 PV module is a thin-film PV module, and it is made up of copper selenide. The short-circuit current of any PV module during different operating conditions can be computed by Eq. 23 .

$I_{S C}(G, T)=I_{S C(S T C)} \times \frac{G}{G_{S T C}}+\alpha\left(T-T_{S T C}\right)$

where $G_{S T C}$ and $T_{S T C}$ denote the irradiation and temperature at standard testing conditions (STC), $G$ and $T$ represent the actual irradiation and temperature, $\alpha$ denotes the temperature coefficient, and $I_{s c(S T C)}$ represents the short-circuit current under STC.

The proposed OLBGWO algorithm is directly applied to this commercial PV module to validate the performance. The experimental samples are collected for two different conditions, such as constant temperature, i.e., at $25{ }^{\circ} \mathrm{C}$ and variable irradiations, i.e., at $200 \mathrm{~W} / \mathrm{m}^{2}, 400 \mathrm{~W} / \mathrm{m}^{2}, 600$ $\mathrm{W} / \mathrm{m}^{2}, 800 \mathrm{~W} / \mathrm{m}^{2}$, and $1000 \mathrm{~W} / \mathrm{m}^{2}$, and constant irradiation, i.e., at $1000 \mathrm{~W} / \mathrm{m}^{2}$ and variable temperature, i.e., at $25{ }^{\circ} \mathrm{C}, 40{ }^{\circ} \mathrm{C}, 50{ }^{\circ} \mathrm{C}$, and $70{ }^{\circ} \mathrm{C}$. The parameters optimized by the proposed 
OLBGWO algorithm are listed in Table 12 and Table 13, respectively, for both test cases. Table 12-13 display that the OLBGWO algorithm precisely estimates the parameters of the PV module. To visualize the identical curve between the experimental value and simulated value, I-V characteristics for both test cases are illustrated in Fig. 13 and Fig. 14, respectively.

Table 12. Optimized parameters at $25^{\circ} \mathrm{C}$ temperature - ST40 PV module

\begin{tabular}{|c|c|c|c|c|c|c|}
\hline Irradiation & $\boldsymbol{I}_{\boldsymbol{p}}(\boldsymbol{A})$ & $\boldsymbol{a}$ & $\boldsymbol{R}_{\boldsymbol{s h}}(\mathbf{\Omega})$ & $\boldsymbol{R}_{\boldsymbol{s e}}(\mathbf{\Omega})$ & $\boldsymbol{I}_{\boldsymbol{s d}}(\boldsymbol{A})$ & $\boldsymbol{R M S E}$ \\
\hline $\mathbf{1 0 0 0} \mathbf{W} / \mathbf{m}^{\mathbf{2}}$ & 2.6763 & 1.7628 & 355.9927 & 1.1040 & $1.6950 \mathrm{e}-06$ & $9.5666 \mathrm{e}-04$ \\
\hline $\mathbf{8 0 0} \mathbf{W} / \mathbf{m}^{\mathbf{2}}$ & 2.1337 & 1.7682 & 393.2737 & 1.0931 & $1.7422 \mathrm{e}-06$ & 0.0017 \\
\hline $\mathbf{6 0 0} \mathbf{W} / \mathbf{m}^{\mathbf{2}}$ & 1.6049 & 1.7426 & 346.6038 & 1.1155 & $1.4121 \mathrm{e}-06$ & $6.7540 \mathrm{e}-04$ \\
\hline $\mathbf{4 0 0} \mathbf{W} / \mathbf{m}^{2}$ & 1.0662 & 1.8109 & 385.5736 & 1.0405 & $2.3474 \mathrm{e}-06$ & $8.1112 \mathrm{e}-04$ \\
\hline $\mathbf{2 0 0} \mathbf{W} / \mathbf{m}^{\mathbf{2}}$ & 0.5328 & 1.7952 & 348.3370 & 0.9816 & $2.0292 \mathrm{e}-06$ & $5.3039 \mathrm{e}-04$ \\
\hline
\end{tabular}

Table 13. Optimized parameters at $1000 \mathrm{~W} / \mathrm{m}^{2}$ irradiation - ST40 PV module

\begin{tabular}{|c|c|c|c|c|c|c|}
\hline Temperature & $\boldsymbol{I}_{\boldsymbol{p}}(\boldsymbol{A})$ & $\boldsymbol{a}$ & $\boldsymbol{R}_{\boldsymbol{s h}}(\mathbf{\Omega})$ & $\boldsymbol{R}_{\boldsymbol{s e}}(\mathbf{\Omega})$ & $\boldsymbol{I}_{\boldsymbol{s} \boldsymbol{d}}(\boldsymbol{A})$ & $\boldsymbol{R M S E}$ \\
\hline $\mathbf{2 5}^{\mathbf{}} \mathbf{C}$ & 2.6757 & 1.7605 & 362.4503 & 1.1070 & $1.6628 \mathrm{e}-06$ & $8.1983 \mathrm{e}-04$ \\
\hline $\mathbf{4 0}^{\circ} \mathbf{C}$ & 2.6796 & 1.7288 & 383.2375 & 1.1263 & $5.9453 \mathrm{e}-06$ & 0.0014 \\
\hline $\mathbf{5 0}^{\circ} \mathbf{C}$ & 2.6921 & 1.7141 & 292.1694 & 1.1517 & $1.8227 \mathrm{e}-05$ & 0.0018 \\
\hline $\mathbf{7 0}^{\circ} \mathbf{C}$ & 2.6923 & 1.7273 & 367.7540 & 1.1259 & $8.7521 \mathrm{e}-05$ & $7.7772 \mathrm{e}-04$ \\
\hline
\end{tabular}



Figure 13. I-V characteristics of ST40 at $25^{\circ} \mathrm{C}$ and different irradiation 


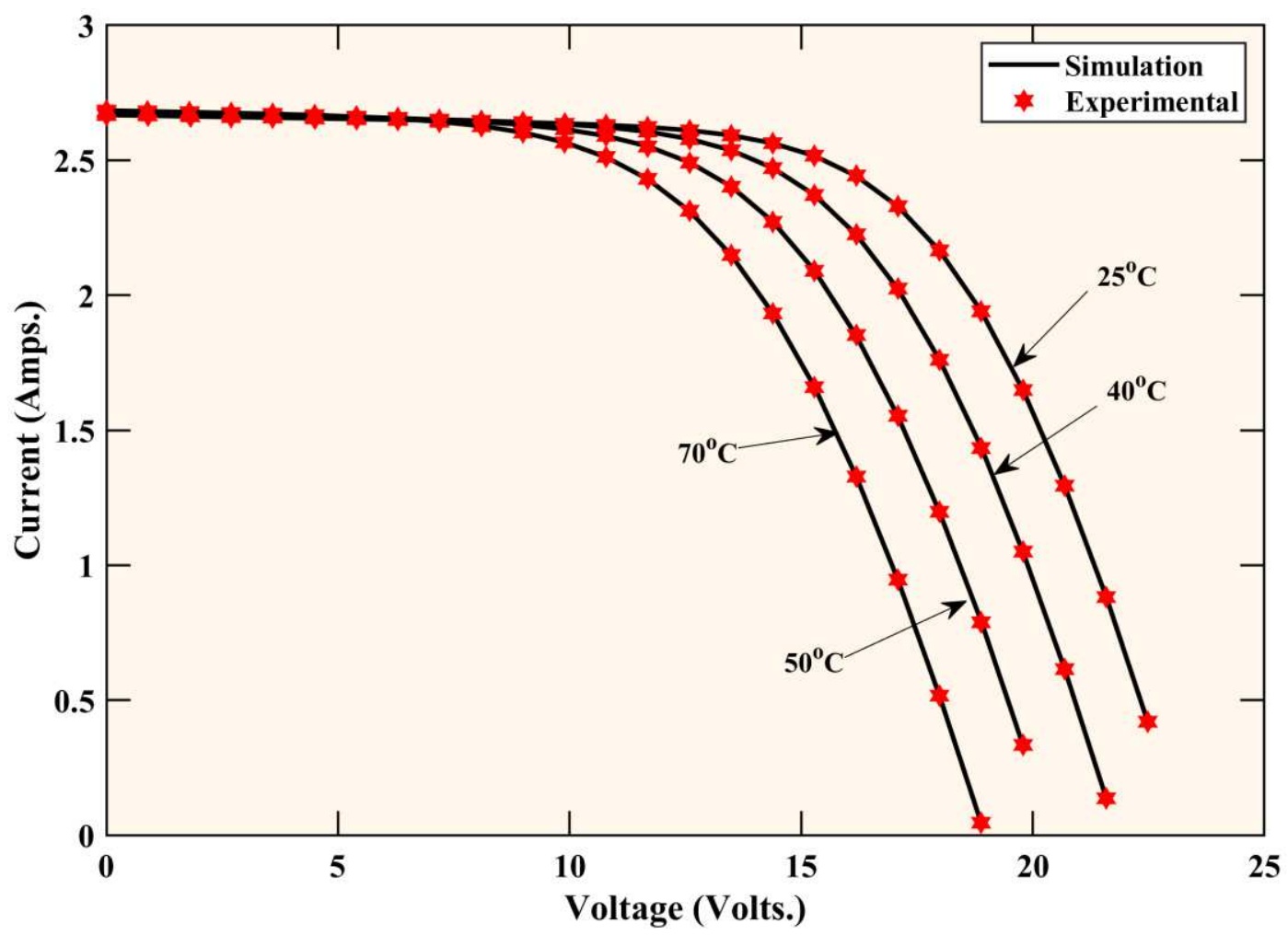

Figure 14. I-V characteristics of ST40 at $1000 \mathrm{~W} / \mathrm{m}^{2}$ and different temperature

From the obtained results, it is noticed that the I-V curves plotted using the estimated variables are steady with the experimental value data at different irradiation and the temperature ranges, and the proposed OLBGWO can attain a low RMSE value. From these discussions, it is clear that the efficiency of the suggested OLBGWO algorithm is reliable and stable in handling the dynamic change in operating conditions. From the above-all discussion, it is concluded that the proposed OLBGWO is a robust tool for identifying the parameters of the commercial PV module.

\subsection{Case-4: Commercial KC200GT PV Module}

To further confirm the efficiency, the OLBGWO algorithm is also examined while optimizing the parameters of the commercial KC200GT PV module. The experimental values of the KC200GT commercial module under different irradiance and temperature conditions are collected to confirm the efficiency of the OLBGWO algorithm. The KC200GT PV module is a multi-crystalline commercial PV module. The proposed OLBGWO algorithm is directly applied to this commercial PV module to validate the performance. The experimental samples are 
collected for two different conditions, such as constant temperature, i.e., at $25{ }^{\circ} \mathrm{C}$ and variable irradiations, i.e., at $200 \mathrm{~W} / \mathrm{m}^{2}, 400 \mathrm{~W} / \mathrm{m}^{2}, 600 \mathrm{~W} / \mathrm{m}^{2}, 800 \mathrm{~W} / \mathrm{m}^{2}$, and $1000 \mathrm{~W} / \mathrm{m}^{2}$, and constant irradiation, i.e., at $1000 \mathrm{~W} / \mathrm{m}^{2}$ and variable temperature, i.e., at $25^{\circ} \mathrm{C}, 50{ }^{\circ} \mathrm{C}$, and $75^{\circ} \mathrm{C}$. The parameters optimized by the proposed OLBGWO algorithm are listed in Table 14 and Table 15, respectively, for both test cases. Table 14 and Table 15 show that the OLBGWO algorithm precisely estimates the variables of the PV module. To visualize the identical curve between the experimental value and simulated value, I-V characteristics for both test cases are illustrated in Fig. 15 and Fig. 16, respectively.

Table 14. Optimized parameters at $25^{\circ} \mathrm{C}$ temperature - KC200GT PV module

\begin{tabular}{|c|c|c|c|c|c|c|}
\hline Irradiation & $\boldsymbol{I}_{p}(\boldsymbol{A})$ & $\boldsymbol{a}$ & $\boldsymbol{R}_{\boldsymbol{s} \boldsymbol{}}(\mathbf{\Omega})$ & $\boldsymbol{R}_{s e}(\mathbf{\Omega})$ & $\boldsymbol{I}_{s d}(\boldsymbol{A})$ & $\boldsymbol{R M S E}$ \\
\hline $\mathbf{1 0 0 0} \mathbf{W} / \mathbf{m}^{2}$ & 8.2097 & 1.2477 & $4.8684 \mathrm{e}+03$ & 0.2863 & $4.7620 \mathrm{e}-08$ & 0.0248 \\
\hline $\mathbf{8 0 0} \mathbf{~ W / m ^ { 2 }}$ & 6.5646 & 1.1691 & $3.8246 \mathrm{e}+03$ & 0.3016 & $1.3066 \mathrm{e}-08$ & 0.0169 \\
\hline $\mathbf{6 0 0} \mathbf{W} / \mathbf{m}^{\mathbf{2}}$ & 4.9317 & 1.2459 & $5.0000 \mathrm{e}+03$ & 0.2703 & $4.2656 \mathrm{e}-08$ & 0.0151 \\
\hline $\mathbf{4 0 0} \mathbf{~ W / m ^ { 2 }}$ & 3.2776 & 1.1927 & $4.9867 \mathrm{e}+03$ & 0.2431 & $1.8396 \mathrm{e}-08$ & 0.0080 \\
\hline $\mathbf{2 0 0} \mathbf{~ W / m ^ { 2 }}$ & 1.6452 & 1.1318 & 774.3192 & 0.1111 & $6.5410 \mathrm{e}-09$ & 0.0034 \\
\hline
\end{tabular}

Table 15. Optimized parameters at $1000 \mathrm{~W} / \mathrm{m}^{2}$ irradiation - ST40 PV module

\begin{tabular}{|c|c|c|c|c|c|c|}
\hline Temperature & $\boldsymbol{I}_{p}(\boldsymbol{A})$ & $\boldsymbol{a}$ & $\boldsymbol{R}_{\boldsymbol{s} h}(\mathbf{\Omega})$ & $\boldsymbol{R}_{\boldsymbol{s e}}(\mathbf{\Omega})$ & $\boldsymbol{I}_{\text {sd }}(\boldsymbol{A})$ & $\boldsymbol{R M S E}$ \\
\hline $\mathbf{2 5}^{\mathbf{}} \mathbf{C}$ & 8.2052 & 1.1695 & $3.4094 \mathrm{e}+03$ & 0.3132 & $1.3195 \mathrm{e}-08$ & 0.0136 \\
\hline $\mathbf{5 0}^{\mathbf{}} \mathbf{C}$ & 8.2880 & 1.1515 & $4.9186 \mathrm{e}+03$ & 0.3260 & $2.1583 \mathrm{e}-07$ & 0.0063 \\
\hline $\mathbf{7 5}^{\circ} \mathbf{C}$ & 8.3717 & 1.1141 & $1.4679 \mathrm{e}+03$ & 0.3394 & $1.9443 \mathrm{e}-06$ & 0.0052 \\
\hline
\end{tabular}




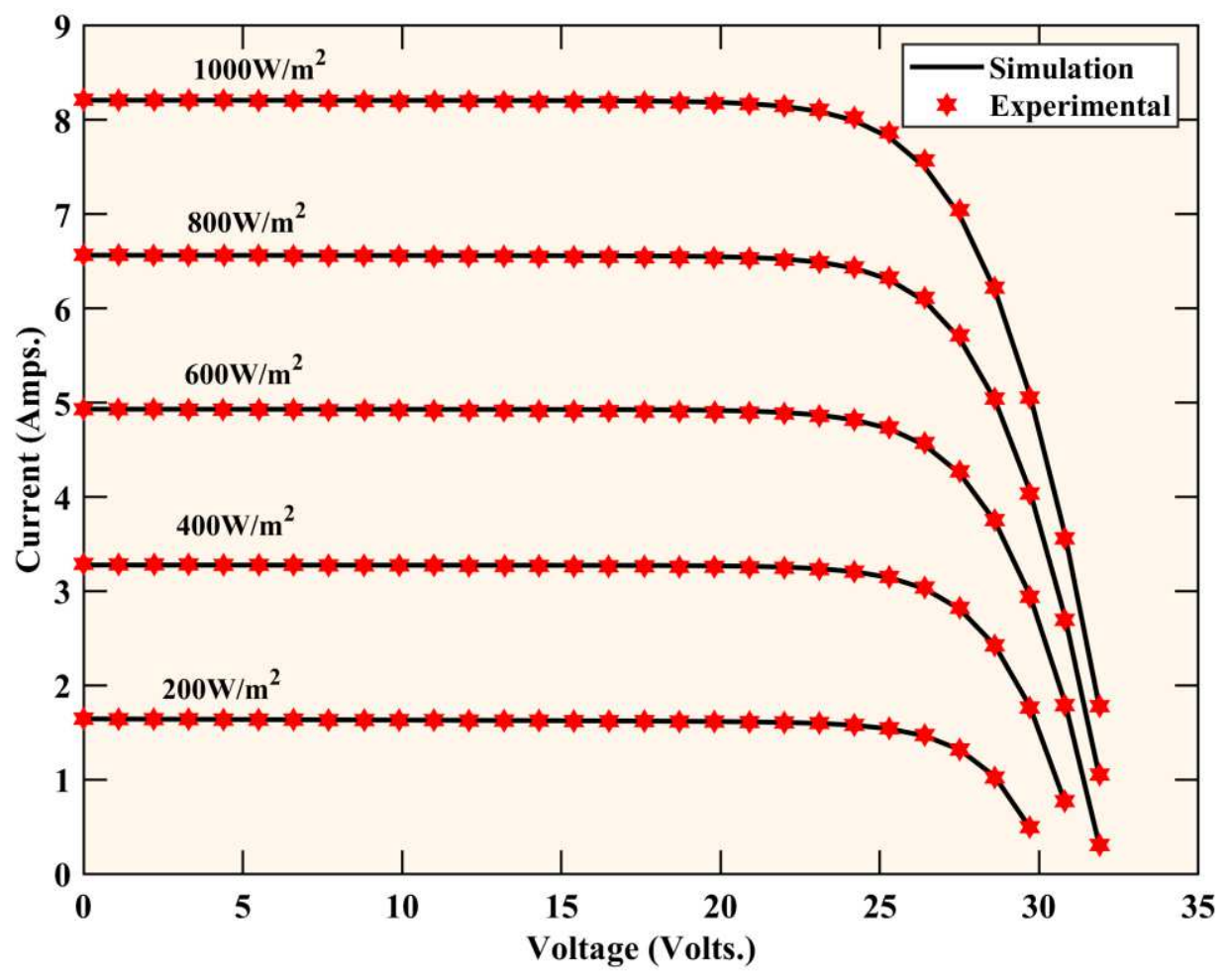

Figure 15. I-V characteristics of KC200GT at $25{ }^{\circ} \mathrm{C}$ and different irradiation

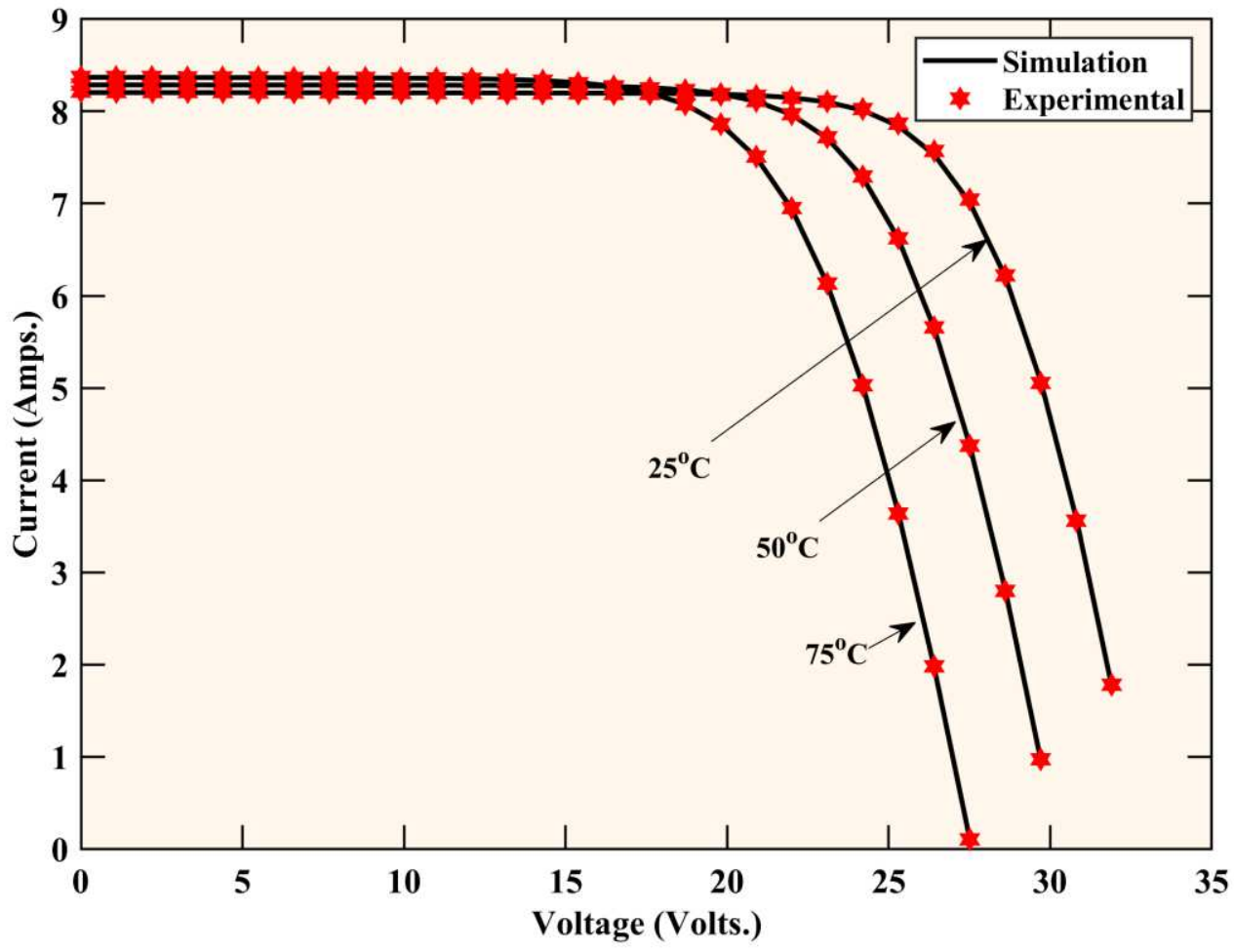

Figure 16. I-V curves of KC200GT at $1000 \mathrm{~W} / \mathrm{m}^{2}$ and different temperature 
From the obtained results, it is noticed that the I-V curves plotted using the estimated parameters are steady with the experimental value data at different irradiation and the temperature ranges, and the proposed OLBGWO can attain a low RMSE value. From these discussions, it is clear that the efficiency of the suggested OLBGWO algorithm is reliable and stable in handling the dynamic change in operating conditions.

\subsection{Statistical Test}

In order to prove the superiority of the OLBGWO algorithm in terms of the overall ranking, the Wilcoxon signed-rank test (WRT) was carried out. A detailed comparison is made among different algorithms, such as OLBGWO, GWO, HHO, SSA, SMA, SCA, PSO, and MFO. The WRT has been carried out at a significant level of 0.05 to examine the performance for two test cases (Case-1 and Case-2). The WRT is a nonparametric test, and this test is used to find the statistical difference between the two techniques. The results for two different cases, such as Cae-1 and Case-2, are listed in Table 16 and Table 17, respectively. Finally, it is concluded that the OLBGWO algorithm performs better than other selected algorithms.

Table 16. Case-1: Statistical WRT results

\begin{tabular}{|c|c|c|c|}
\hline OLBGWO Vs & $R+$ & $R$ - & $p$-value \\
\hline \multicolumn{4}{|c|}{ SDM } \\
\hline SMA & 10 & 11 & 1 \\
\hline GWO & 15 & 6 & 0.1 \\
\hline PSO & 15 & 6 & 0.1 \\
\hline SCA & 15 & 6 & 0.1 \\
\hline SSA & 15 & 6 & 0.1 \\
\hline HHO & 13 & 8 & 0.4 \\
\hline MFO & 15 & 6 & 0.1 \\
\hline SMA & 10 & 11 & 1 \\
\hline GWO & 15 & 6 & 0.1 \\
\hline PSO & 15 & 6 & 0.1 \\
\hline SCA & 15 & 6 & 0.1 \\
\hline SSA & 15 & 6 & 0.1 \\
\hline HHO & 15 & 6 & 0.1 \\
\hline & 15 & 6 & 0.1 \\
\hline
\end{tabular}


Table 17. Case-2: Statistical WRT results

\begin{tabular}{|c|c|c|c|}
\hline OLBGWO Vs & $\boldsymbol{R +}$ & $\boldsymbol{R}$ - & $\boldsymbol{p}$-value \\
\hline \multicolumn{4}{|c|}{ SDM } \\
\hline SMA & 3 & 3 & 1 \\
\hline GWO & 6 & 0 & 0.2500 \\
\hline PSO & 6 & 0 & 0.2500 \\
\hline SCA & 6 & 0 & 0.2500 \\
\hline SSA & 6 & 0 & 0.2500 \\
\hline HHO & 3 & 3 & 1 \\
\hline MFO & 6 & 0 & 0.2500 \\
\hline \multicolumn{5}{|c|}{ DDM } \\
\hline SMA & 5 & 1 & 0.5000 \\
\hline GWO & 6 & 0 & 0.2500 \\
\hline PSO & 6 & 0 & 0.2500 \\
\hline SCA & 6 & 0 & 0.2500 \\
\hline SSA & 6 & 0 & 0.2500 \\
\hline HHO & 6 & 0 & 0.2500 \\
\hline MFO & 6 & 0 & 0.2500 \\
\hline
\end{tabular}

\subsection{Further Discussions}

In this study, in order to minimize the early convergence of the GWO algorithm and improve its exploitation and exploration abilities, a productive scheme called OLB is integrated with the basic GWO. This new, updated GWO-based approach is used for PV cell and module parameter estimation. This can be inferred that the OLBGWO algorithm can reliably and effectively calculate the uncertain variables for the PV model based on the experimental outcomes of common SDM, DDM, and PV models and two commercial modules derived from the manufacturer's datasheets. The proposed OLBGWO algorithm behaves highly competitive in contrast to other techniques; this finding can be due to the fact that the perception of equilibrium between the primary GWO's exploitation and exploration ability is held in a more acceptable state. Several variables contribute towards a more efficient result in identifying parameters of PV models at different temperature and irradiation settings.

As discussed earlier, for estimation methods of SDM, DDM, and PV models, respectively, the developed OLBGWO algorithm has been used. From the I-V characteristics, it can be concluded that the results reported by OLBGWO agree with all experimental data. The proposed OLBGWO algorithm has better exploitation and exploration abilities than other competitors, including RMSE, convergence speed, and the extraction of best parameters. All the above-said features are 
improved by the OLB strategy and modified vector parameter. The modified vector parameter $a$ improves GWO's local search ability. It guarantees OLBGWO's performance in seeking the best solution with superior potential in the convergence phase. The OLB approach directs the weaker agent to better search space for getting a better convergence curve.

In addition, two practical datasets taken from the datasheets provided by the manufacturers, including KC200GT and ST40, were considered to evaluate the efficiency of the OLBGWO algorithm for handling the complex non-linear problems at various temperatures and irradiation. In the lower temperature, the parameters obtained by the OLBGWO are still compatible with the real information of KC200GT and ST40, and the proposed OLBGWO can still find the less RSME values. These findings can be related to OLBGWO's steadiness. Through the OLB and modified vector parameter process, the efficiency of the basic version of GWO in parameter estimation of the photovoltaic model is improved. The proposed OLBGWO algorithm can thus be considered the most promising approach for estimating the parameters.

\section{Conclusions}

An enhanced GWO algorithm called OLBGWO is proposed in this paper to extract the unknown parameters of various PV models reliably and efficiently. The modified vector parameter and OLB approaches are combined with the basic version of GWO in the OLBGWO to improve its search efficiency. The modified vector parameter can stimulate the ability to exploit and increase the solution accuracy, and the OLB increases the ability to explore and enable the weaker population to escape optimally from the local target. The findings suggest that the proposed OLBGWO algorithm shows a superior performance relative to the other state-of-the-art algorithms based on the convergence speed and solution accuracy.

Although the OLBGWO algorithm demonstrates reasonable success in solving the parameter estimation problem, the control parameter for the OLB method is fixed. Therefore, the suggested OLBGWO algorithm is not a universal parameter-free algorithm. Therefore, future research works are planned as follows: firstly, an adaptive parameter may be introduced in the OLBGWO algorithm to solve real-world problems without any additional parameters. Real-time optimization problems, such as unit commitment, controller tuning, feature selection, economic 
load dispatch, image segmentation, multiobjective problems, etc., may be handled by developing the constrained and binary alternatives of the OLGWO algorithm.

\section{References}

[1] M. Premkumar, U. Subramaniam, T. S. Babu, R. M. Elavarasan, and L. Mihet-Popa, "Evaluation of Mathematical Model to Characterize the Performance of Conventional and Hybrid PV Array Topologies under Static and Dynamic Shading Patterns," Energies, vol. 13, no. 12, pp. 3216, Jun. 2020.

[2] M. Premkumar and R. Sowmya, "Certain Study on MPPT Algorithms to track the Global MPP under Partial Shading on Solar PV Module/Array,” International Journal of Computing and Digital Systems, vol. 8, no. 04, pp. 405-416, Jul. 2019.

[3] Gul M, Kotak Y, and Muneer T., "Review on recent trend of solar photovoltaic technology," Energy Exploration \& Exploitation, vol. 34, no. 4, pp. 485-526, 2016.

[4] Hussin, Farihahusnah, Issabayeva, Gulnaziya and Aroua, Mohamed Kheireddine. "Solar photovoltaic applications: opportunities and challenges" Reviews in Chemical Engineering, vol. 34, no. 4, 2018, pp. 503-528. https://doi.org/10.1515/revce-20160058

[5] P. Manoharan et al., "Improved Perturb \& Observation Maximum Power Point Tracking Technique for Solar Photovoltaic Power Generation Systems," in IEEE Systems Journal, DOI: 10.1109/JSYST.2020.3003255.

[6] Premkumar, M, Ibrahim Mohamed, A, Mohan Kumar, R, and Sowmya, R, "Analysis and Simulation of Bio-Inspired Intelligent Salp Swarm MPPT Method for the PV Systems under Partial Shaded Conditions, " International Journal of Computing and Digital Systems, vol. 8, no. 05, pp. 489-496, Sep. 2019.

[7] Ali Kareem Abdulrazzaq, György Bognár, Balázs Plesz, Accurate method for PV solar cells and modules parameters extraction using I-V curves, Journal of King Saud University - Engineering Sciences, 2020. https://doi.org/10.1016/j.jksues.2020.07.008

[8] Al-Dahidi, Sameer \& Ayadi, Osama \& Adeeb, Jehad \& Alrbai, Mohammad \& Qawasmeh, Bashar. (2018). Extreme Learning Machines for Solar Photovoltaic Power Predictions. Energies. 11. 2725. 10.3390/en11102725. 
[9] Oliva, D., Abd El Aziz, M., \& Ella Hassanien, A. (2017). Parameter estimation of photovoltaic cells using an improved chaotic whale optimization algorithm. Applied Energy, 200, 141-154. https://doi.org/10.1016/j.apenergy.2017.05.029

[10] M. Premkumar, C. Kumar, and R. Sowmya, "Mathematical Modelling of Solar Photovoltaic Cell/Panel/Array based on the Physical Parameters from the Manufacturer's Datasheet," International Journal of Renewable Energy Development, vol. 9, no. 1, pp. 7-22, Feb. 2020. https://doi.org/10.14710/ijred.9.1.7-22

[11] Humada, Ali M. \& Hojabri, Mojgan \& Mekhilef, Saad \& Hamada, Hussein M., 2016. "Solar cell parameters extraction based on single and double-diode models: A review," Renewable and Sustainable Energy Reviews, Elsevier, vol. 56(C), pages 494-509.

[12] Ramzi Ben Messaoud, Extraction of uncertain parameters of single-diode model of a photovoltaic panel using simulated annealing optimization, Energy Reports, Volume 6, 2020, Pages 350-357.

[13] Ramadan, A., Kamel, S., Korashy, A. et al. Photovoltaic Cells Parameter Estimation Using an Enhanced Teaching-Learning-Based Optimization Algorithm. Iran J Sci Technol Trans Electr Eng 44, 767-779 (2020). https://doi.org/10.1007/s40998-01900257-9

[14] Q. Hao, Z. Zhou, Z. Wei and G. Chen, "Parameters Identification of Photovoltaic Models Using a Multi-Strategy Success-History-Based Adaptive Differential Evolution," in IEEE Access, vol. 8, pp. 35979-35994, 2020, doi: 10.1109/ACCESS.2020.2975078.

[15] T. Easwarakhanthan, J. Bottin, I. Bouhouch \& C. Boutrit (1986) Nonlinear Minimization Algorithm for Determining the Solar Cell Parameters with Microcomputers, International Journal of Solar Energy, 4:1, 1-12, DOI: 10.1080/01425918608909835.

[16] D.S.H. Chan, J.R. Phillips, J.C.H. Phang, A comparative study of extraction methods for solar cell model parameters, Solid-State Electronics, Volume 29, Issue 3, 1986, Pages 329-337.

[17] M Premkumar, R Sowmya, S Umashankar, and J Pradeep, "An Effective Solar Photovoltaic Module Parameter Estimation Technique for Single-Diode Model,” IOP Conference Series: Materials Science and Engineering, Volume 937, Recent Trends on 
Renewable Energy Smart Grid and Electric Vehicle Technologies (RESGEVT 2020) 9 July 2020, Vellore, India

[18] M. Premkumar, P. Jangir, R. Sowmya, H. H. Alhelou, A. A. Heidari and H. Chen, "MOSMA: Multi-Objective Slime Mould Algorithm Based on Elitist Non-Dominated Sorting," in IEEE Access, vol. 9, pp. 3229-3248, 2021, doi: 10.1109/ACCESS.2020.3047936.

[19] Huiling Chen, Qian Zhang, Jie Luo, Yueting Xu, Xiaoqin Zhang, An enhanced Bacterial Foraging Optimization and its application for training kernel extreme learning machine, Applied Soft Computing, Volume 86, 2020, 105884.

[20] Huiling Chen, Mingjing Wang, Xuehua Zhao, A multi-strategy enhanced sine cosine algorithm for global optimization and constrained practical engineering problems, Applied Mathematics and Computation, Volume 369, 2020, 124872.

[21] Yin, Y., Tu, Q. \& Chen, X. Enhanced Salp Swarm Algorithm based on random walk and its application to training feedforward neural networks. Soft Comput 24, 1479114807 (2020). https://doi.org/10.1007/s00500-020-04832-9

[22] Kashif Ishaque, Zainal Salam, Saad Mekhilef, Amir Shamsudin, Parameter extraction of solar photovoltaic modules using penalty-based differential evolution, Applied Energy, Volume 99, 2012, Pages 297-308

[23] Ridha, H.M., Gomes, C. \& Hizam, H. Estimation of photovoltaic module model's parameters using an improved electromagnetic-like algorithm. Neural Comput \& Applic 32, 12627-12642 (2020).

[24] Wu Z, Xie Z, Liu C. An improved lion swarm optimization for parameters identification of photovoltaic cell models. Transactions of the Institute of Measurement and Control. 2020;42(6):1191-1203.

[25] Obiora, V., Saha, C., Bazi, A.A. et al. Optimization of solar photovoltaic (PV) parameters using meta-heuristics. Microsystem Technology (2020). https://doi.org/10.1007/s00542-020-05066-3

[26] Fayrouz Dkhichi, Benyounes Oukarfi, Abderrahim Fakkar, Noureddine Belbounaguia, Parameter identification of solar cell model using Levenberg-Marquardt algorithm combined with imulated annealing, Solar Energy, Volume 110, 2014, Pages 781-788. 
[27] H. Sheng, C. Li, H. Wang, Z. Yan, Y. Xiong, Z. Cao, and Q. Kuang, "Parameters Extraction of Photovoltaic Models Using an Improved Moth-Flame Optimization," Energies, vol. 12, no. 18, p. 3527, Sep. 2019.

[28] N. Krishnakumar, R. Venugopalan and N. Rajasekar, "Bacterial foraging algorithm based parameter estimation of solar PV model," 2013 Annual International Conference on Emerging Research Areas and 2013 International Conference on Microelectronics, Communications and Renewable Energy, Kanjirapally, 2013, pp. 1-6, doi: 10.1109/AICERA-ICMiCR.2013.6575948.

[29] R. Wang, Y. Zhan, and H. Zhou, "Application of Artificial Bee Colony in Model Parameter Identification of Solar Cells," Energies, vol. 8, no. 8, pp. 7563-7581, Jul. 2015.

[30] Alireza Askarzadeh, Alireza Rezazadeh, Artificial bee swarm optimization algorithm for parameters identification of solar cell models, Applied Energy, Volume 102, 2013, Pages 943-949,

[31] Alireza Askarzadeh, Alireza Rezazadeh, Parameter identification for solar cell models using harmony search-based algorithms, Solar Energy, Volume 86, Issue 11, 2012, Pages 3241-3249

[32] B. Subudhi and R. Pradhan, "Bacterial Foraging Optimization Approach to Parameter Extraction of a Photovoltaic Module," in IEEE Transactions on Sustainable Energy, vol. 9, no. 1, pp. 381-389, Jan. 2018, DOI: 10.1109/TSTE.2017.2736060.

[33] Shuhui $\mathrm{Xu}$, Yong Wang, Parameter estimation of photovoltaic modules using a hybrid flower pollination algorithm, Energy Conversion and Management, Volume 144, 2017, Pages 53-68

[34] H.G.G. Nunes, J.A.N. Pombo, P.M.R. Bento, S.J.P.S. Mariano, M.R.A. Calado, Collaborative swarm intelligence to estimate PV parameters, Energy Conversion and Management, Volume 185, 2019, Pages 866-890

[35] Abbassi, Abdelkader \& Abbassi, Rabeh \& Heidari, Ali Asghar \& Oliva, Diego \& Chen, Huiling \& Habib, Arslan \& Jemli, Mohamed \& Wang, Mingjing, 2020. "Parameters identification of photovoltaic cell models using enhanced exploratory salp chains-based approach," Energy, Elsevier, vol. 198(C). 
[36] Dalia Yousri, Dalia Allam, M.B. Eteiba, Ponnuthurai Nagaratnam Suganthan, Static and dynamic photovoltaic models' parameters identification using Chaotic Heterogeneous Comprehensive Learning Particle Swarm Optimizer variants, Energy Conversion and Management, Volume 182, 2019, Pages 546-563

[37] M. Premkumar, Pradeep Jangir, R. Sowmya, Rajvikram Madurai Elavarasan, B. Santhosh Kumar, Enhanced chaotic JAYA algorithm for parameter estimation of photovoltaic cell/ modules, ISA Transactions, 2021. https://doi.org/10.1016/j.isatra.2021.01.045.

[38] Manoharan Premkumar, Ravichandran Sowmya, Subramaniam Umashankar, Pradeep Jangir, Extraction of uncertain parameters of single-diode photovoltaic module using hybrid particle swarm optimization and grey wolf optimization algorithm, Materials Today: Proceedings, 2020. https://doi.org/10.1016/j.matpr.2020.08.784.

[39] Premkumar, M., Babu, T. S., Umashankar, S., and Sowmya, R., "A new metaphor-less algorithms for the photovoltaic cell parameter estimation", Optik, vol. 208, p. 164559, 2020. doi:10.1016/j.jjleo.2020.164559.

[40] Kumar, C., Raj, T. D., Premkumar, M., and Raj, T. D., "A new stochastic slime mould optimization algorithm for the estimation of solar photovoltaic cell parameters", Optik, vol. 223, p. 165277, 2020. doi:10.1016/j.ijleo.2020.165277

[41] M. Premkumar, R. Sowmya, P. Jangir and J. S. V. Siva Kumar, "A New and Reliable Objective Functions for Extracting the Unknown Parameters of Solar Photovoltaic Cell Using Political Optimizer Algorithm," 2020 International Conference on Data Analytics for Business and Industry: Way Towards a Sustainable Economy (ICDABI), Sakheer, Bahrain, 2020, pp. 1-6, DOI: 10.1109/ICDABI51230.2020.9325627.

[42] Mohammed H. Qais, Hany M. Hasanien, Saad Alghuwainem, Identification of electrical parameters for three-diode photovoltaic model using analytical and sunflower optimization algorithm, Applied Energy, Volume 250, 2019, Pages 109-117

[43] Ahmed Fathy, Mohamed Abd Elaziz, Enas Taha Sayed, A.G. Olabi, Hegazy Rezk, Optimal parameter identification of triple-junction photovoltaic panel based on enhanced moth search algorithm, Energy, Volume 188, 2019, 116025

[44] Huiling Chen, Shan Jiao, Ali Asghar Heidari, Mingjing Wang, Xu Chen, Xuehua Zhao, An opposition-based sine cosine approach with local search for parameter estimation of 
photovoltaic models, Energy Conversion and Management, Volume 195, 2019, Pages 927-942

[45] Hongliang Zhang, Ali Asghar Heidari, Mingjing Wang, Lejun Zhang, Huiling Chen, Chengye Li, Orthogonal Nelder-Mead moth flame method for parameters identification of photovoltaic modules, Energy Conversion and Management, Volume 211, 2020, 112764

[46] Dhruv Kler, Yagyadatta Goswami, K.P.S. Rana, Vineet Kumar, A novel approach to parameter estimation of photovoltaic systems using hybridized optimizer, Energy Conversion and Management, Volume 187, 2019, Pages 486-511

[47] Huiling Chen, Shan Jiao, Mingjing Wang, Ali Asghar Heidari, Xuehua Zhao, Parameters identification of photovoltaic cells and modules using diversificationenriched Harris hawks optimization with chaotic drifts, Journal of Cleaner Production, Volume 244, 2020, 118778

[48] Seyedali Mirjalili, Seyed Mohammad Mirjalili, Andrew Lewis, Grey Wolf Optimizer, Advances in Engineering Software, Volume 69, 2014, Pages 46-61

[49] Faris, H., Aljarah, I., Al-Betar, M.A. et al. Grey wolf optimizer: a review of recent variants and applications. Neural Comput \& Applic 30, 413-435 (2018). https://doi.org/10.1007/s00521-017-3272-5

[50] Seyedali Mirjalili, Shahrzad Saremi, Seyed Mohammad Mirjalili, Leandro dos S. Coelho, Multi-objective grey wolf optimizer: A novel algorithm for multi-criterion optimization, Expert Systems with Applications, Volume 47, 2016, Pages 106-119

[51] D. H. Wolpert and W. G. Macready, "No free lunch theorems for optimization," in IEEE Transactions on Evolutionary Computation, vol. 1, no. 1, pp. 67-82, April 1997, DOI: $10.1109 / 4235.585893$.

[52] Manoharan Premkumar, Ravichandran Sowmya, Mohamed I. Mosaad, Tarek A. Abdul Fattah, Design and development of low-cost photovoltaic module characterization educational demonstration tool, Materials Today: Proceedings, 2020. https://doi.org/10.1016/j.matpr.2020.09.135.

[53] Nitin Mittal, Urvinder Singh, and Balwinder Singh Sohi, "Modified Grey Wolf Optimizer for Global Engineering Optimization,” Applied Computational Intelligence and Soft Computing, vol. 2016, pp. 7950348, 2016. 
[54] Qingfu Zhang and Yiu-Wing Leung, "An orthogonal genetic algorithm for multimedia multicast routing," in IEEE Transactions on Evolutionary Computation, vol. 3, no. 1, pp. 53-62, April 1999, DOI: 10.1109/4235.752920.

[55] Guojiang Xiong, Dongyuan Shi, Orthogonal learning competitive swarm optimizer for economic dispatch problems, Applied Soft Computing, Volume 66, 2018, Pages 134148. 
Figures

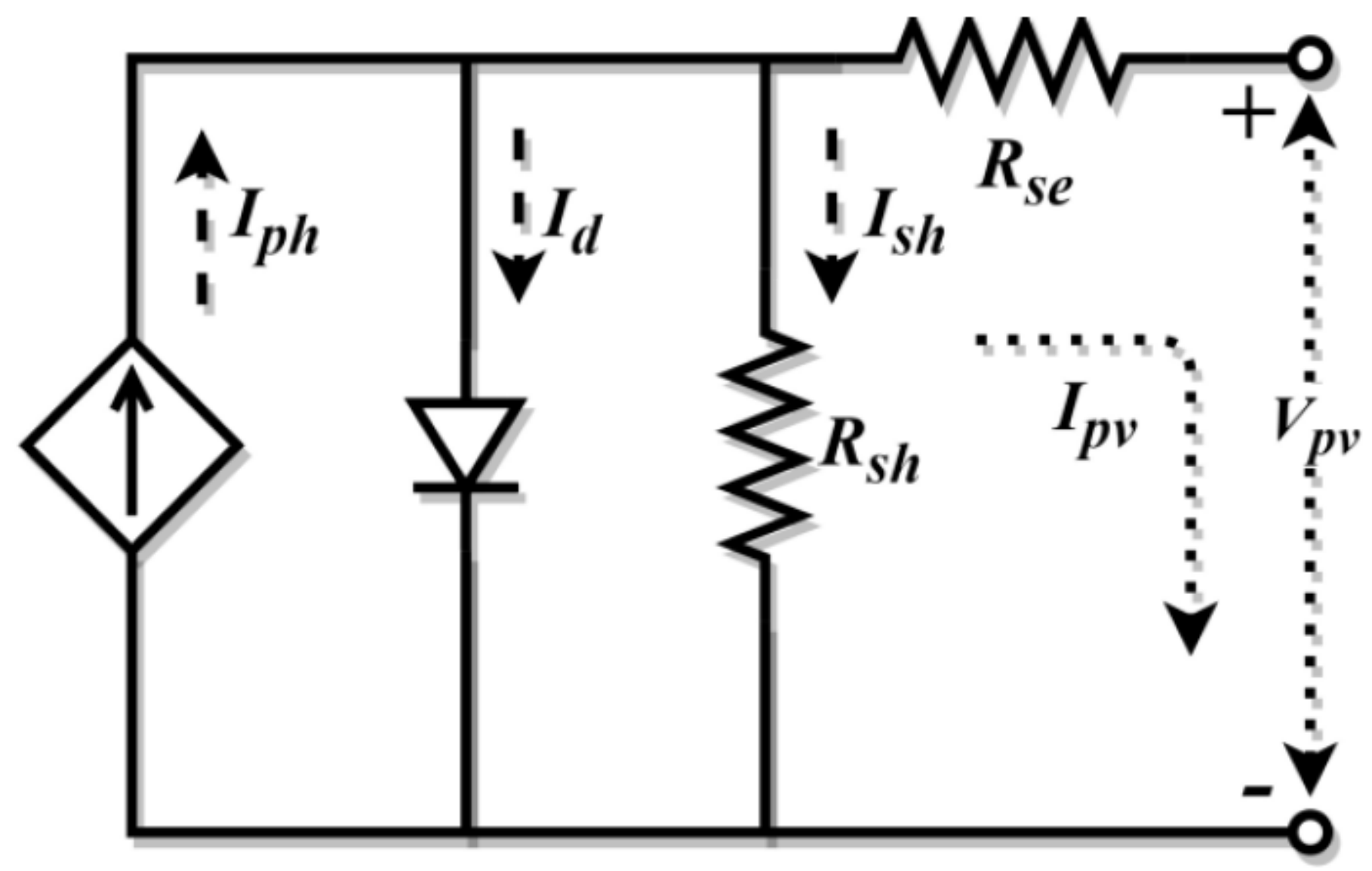

Figure 1

SDM of the solar cell 


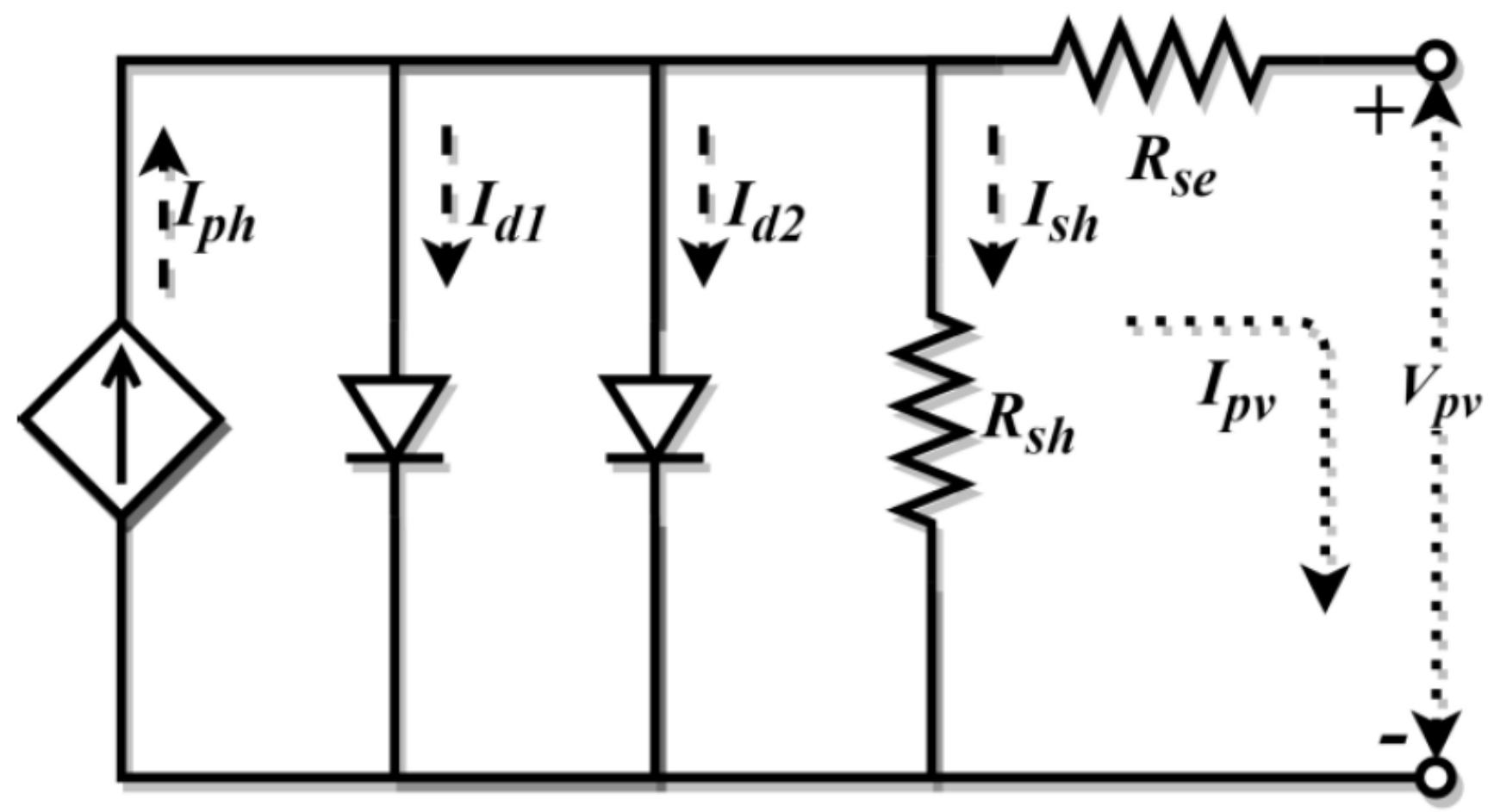

Figure 2

DDM of the solar cell




Figure 3

Electrical circuit of photovoltaic model



Figure 4

Flowchart of the proposed OLBGWO algorithm

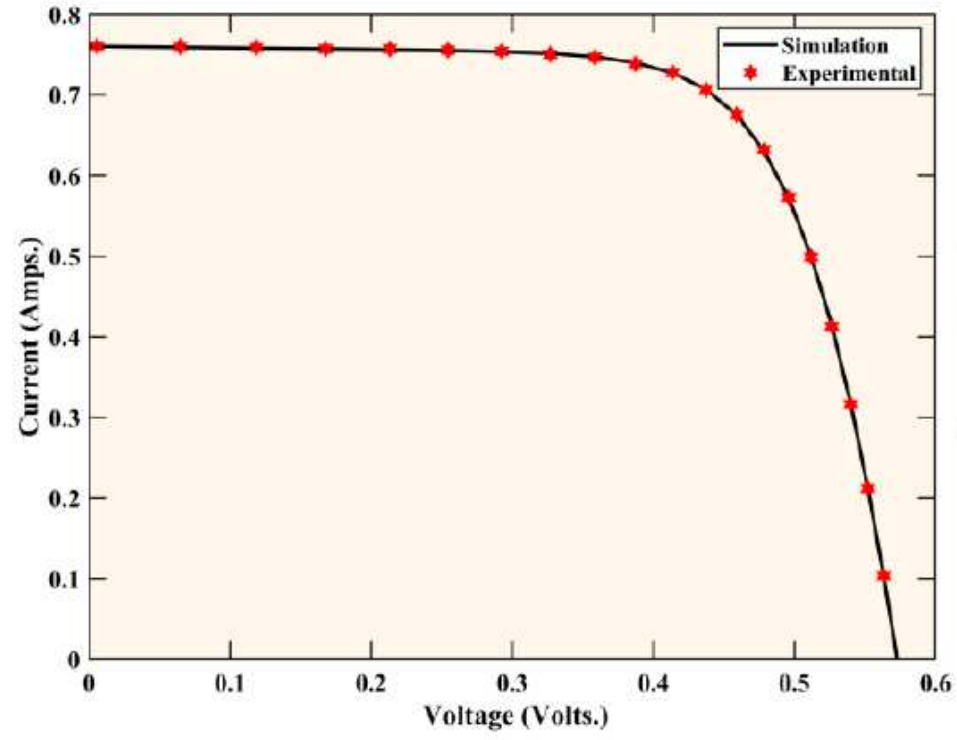

(a)



(b) 
Figure 5

I-V characteristics of the PV cell; (a) SDM, (b) DDM

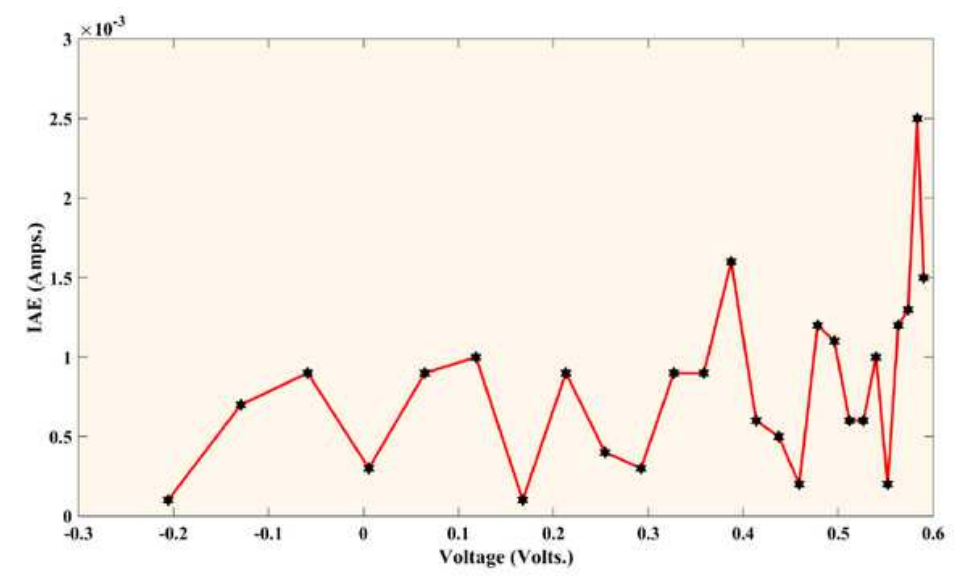

(a)

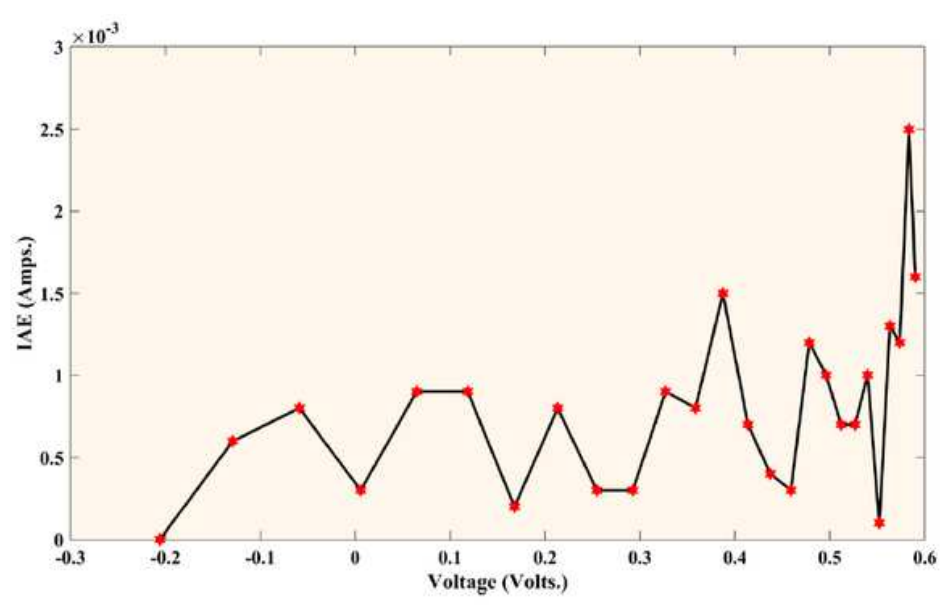

(b)

Figure 6

IAE values of the PV cell; (a) SDM, (b) DDM

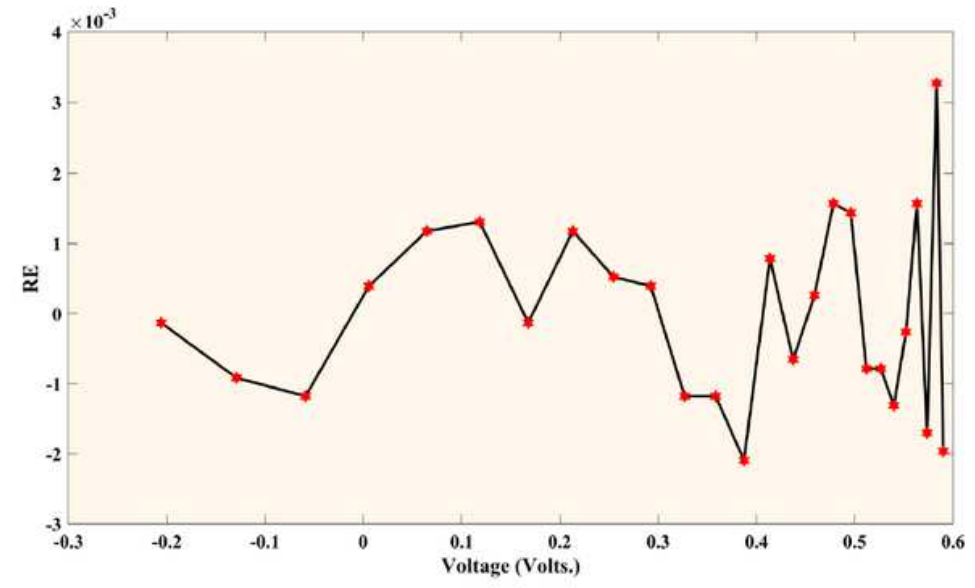

(a)

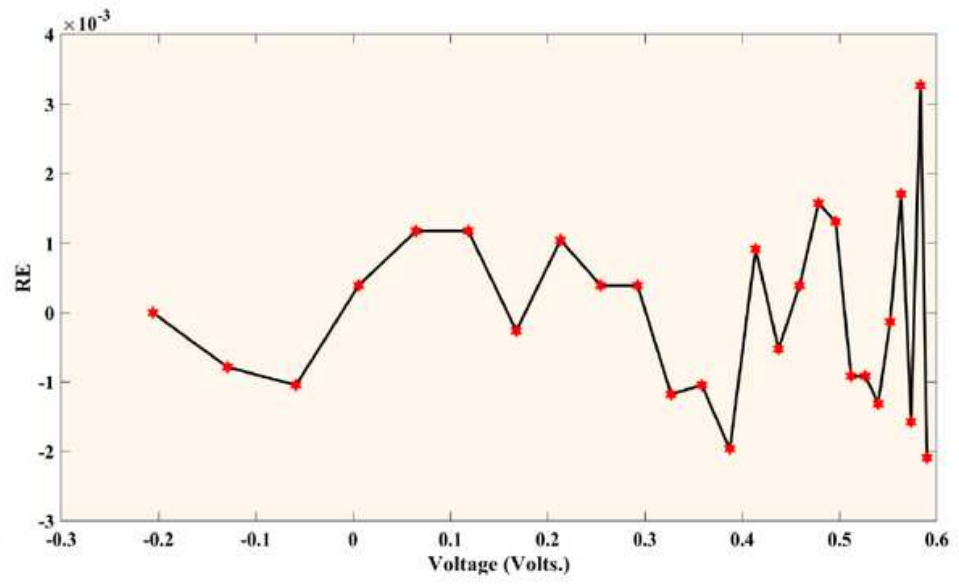

(b)

Figure 7

RE values of the PV cell; (a) SDM, (b) DDM 


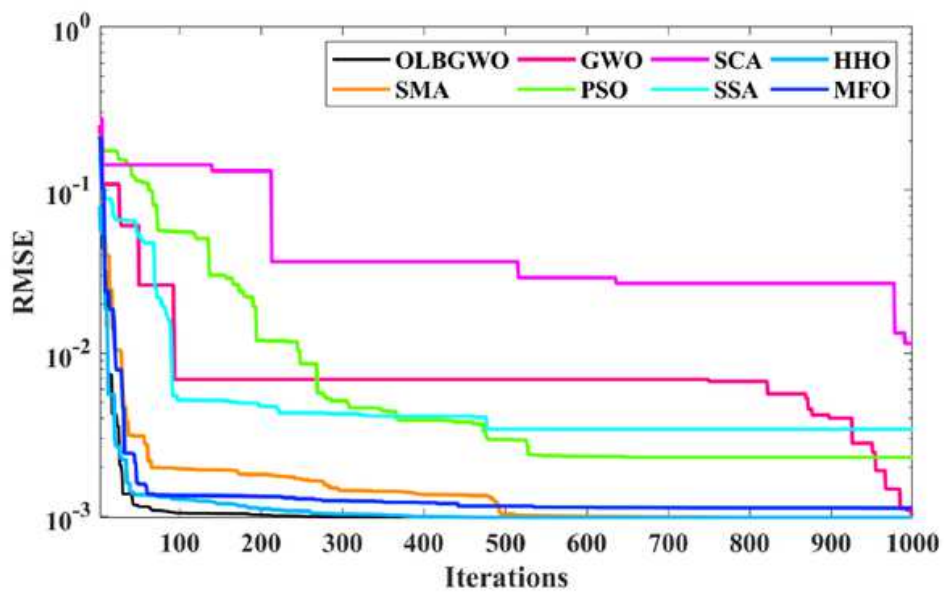

(a)

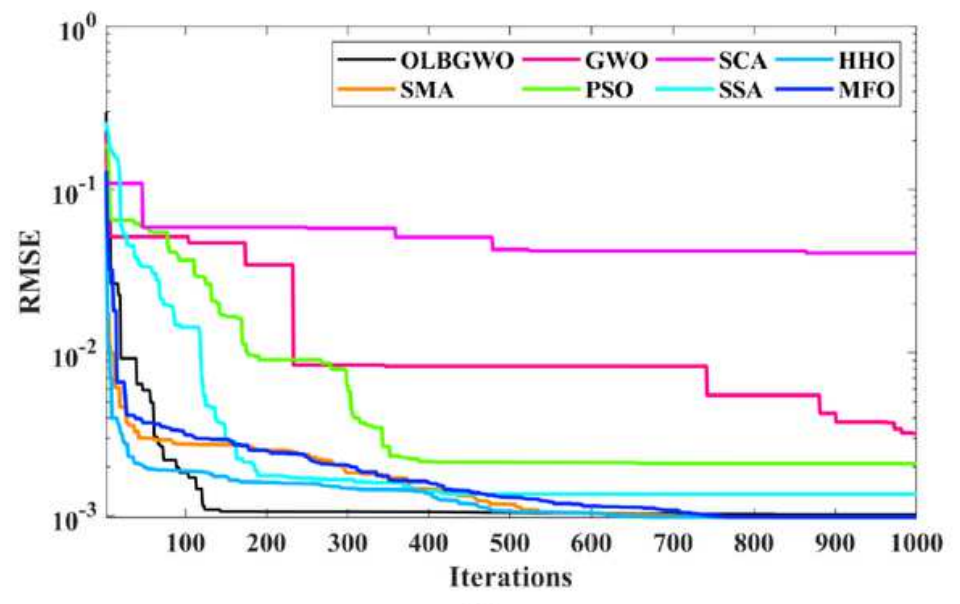

(b)

Figure 8

Convergence curve of all algorithms; (a) SDM, (b) DDM

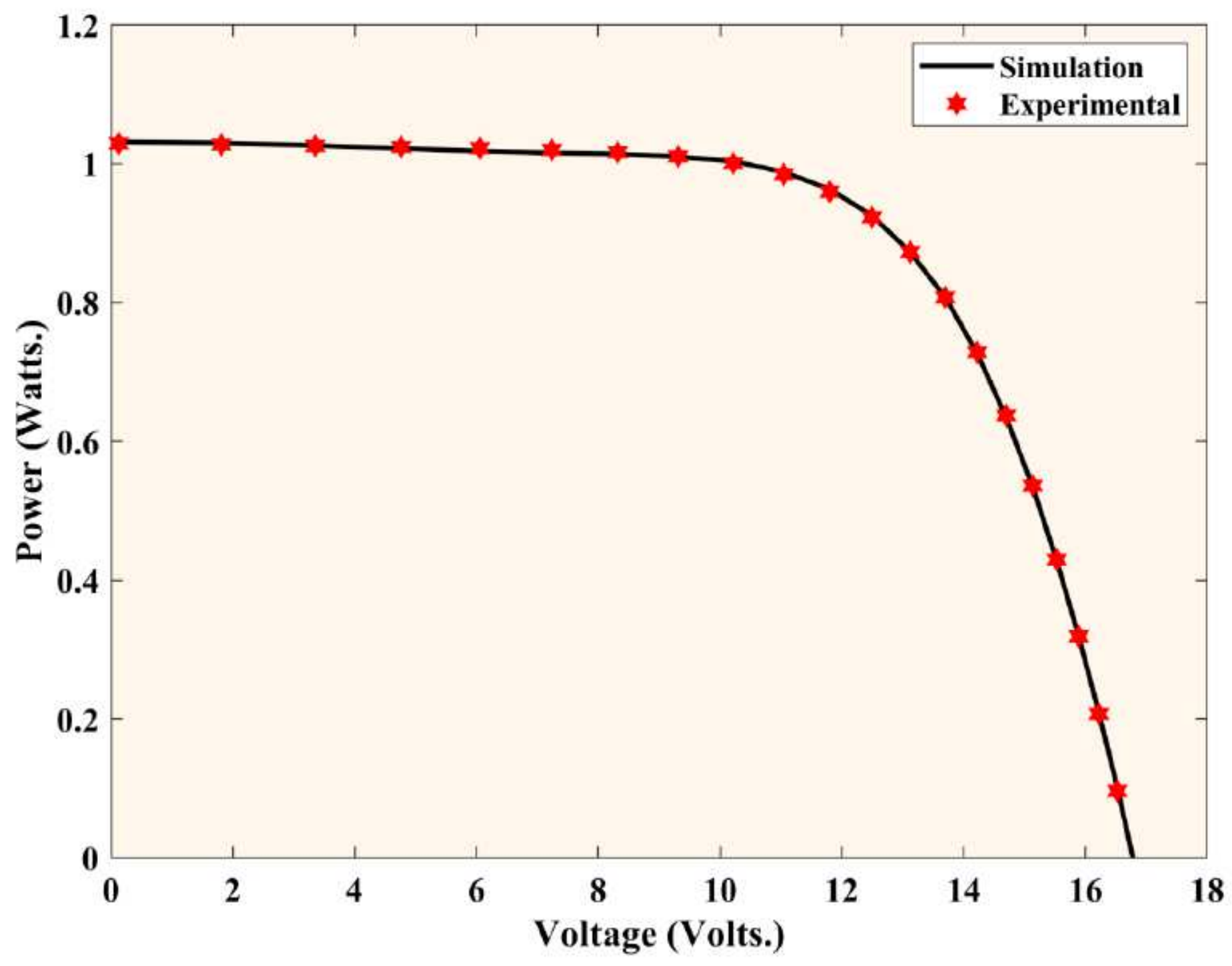

Figure 9 
I-V characteristics of the PV module model

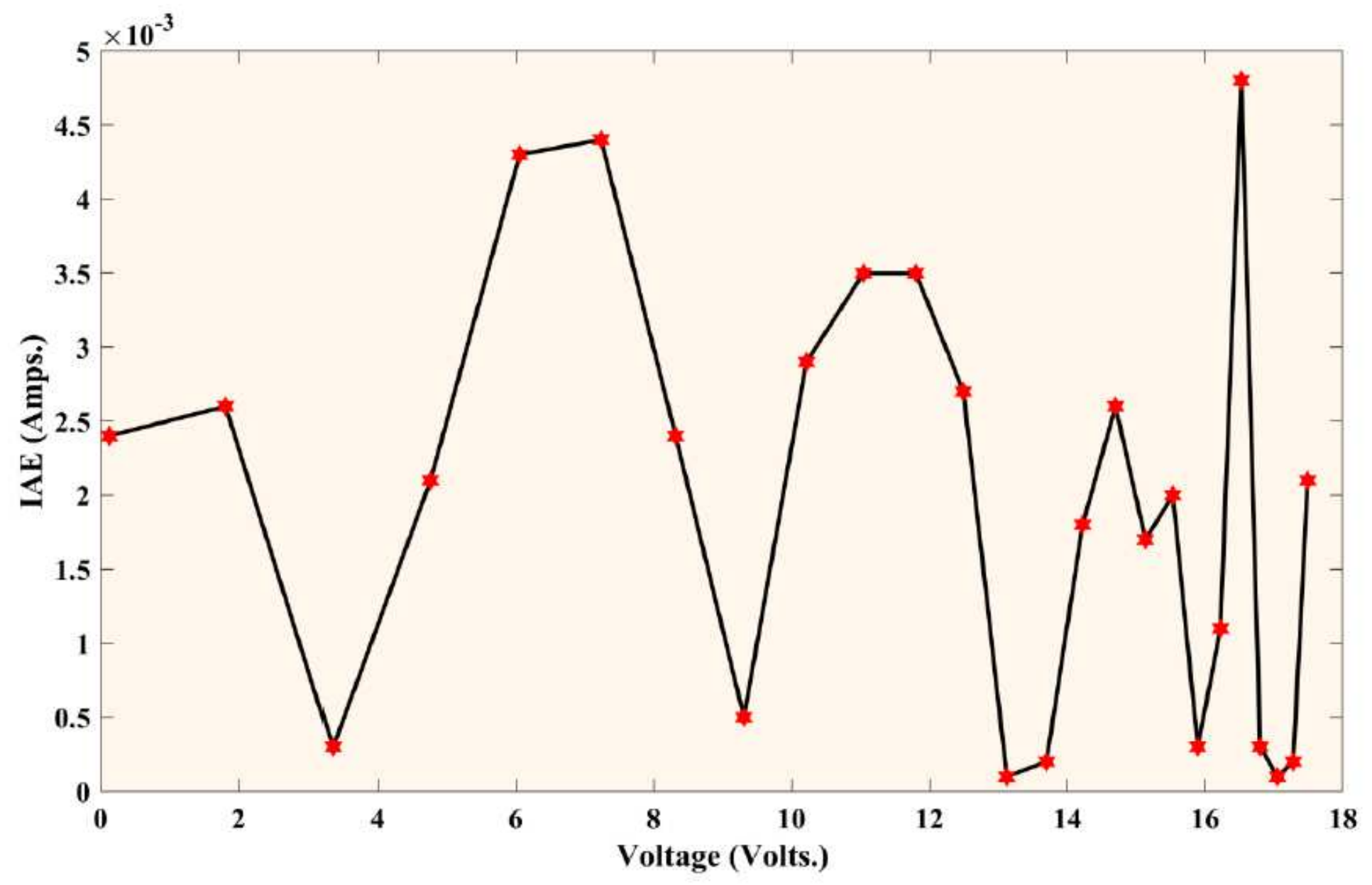

Figure 10

IAE values of the PV module model 


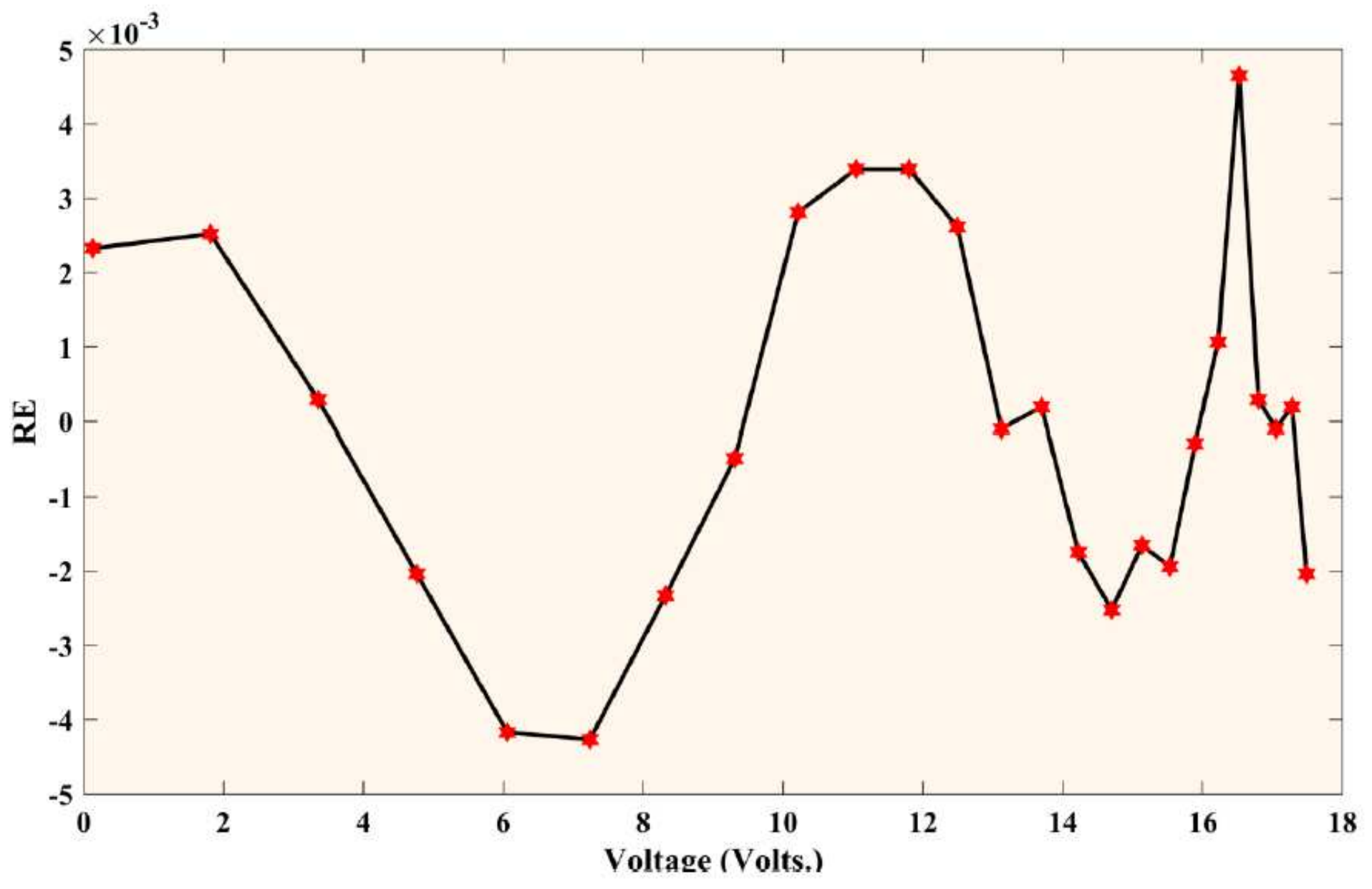

Figure 11

RE values of the PV module model 


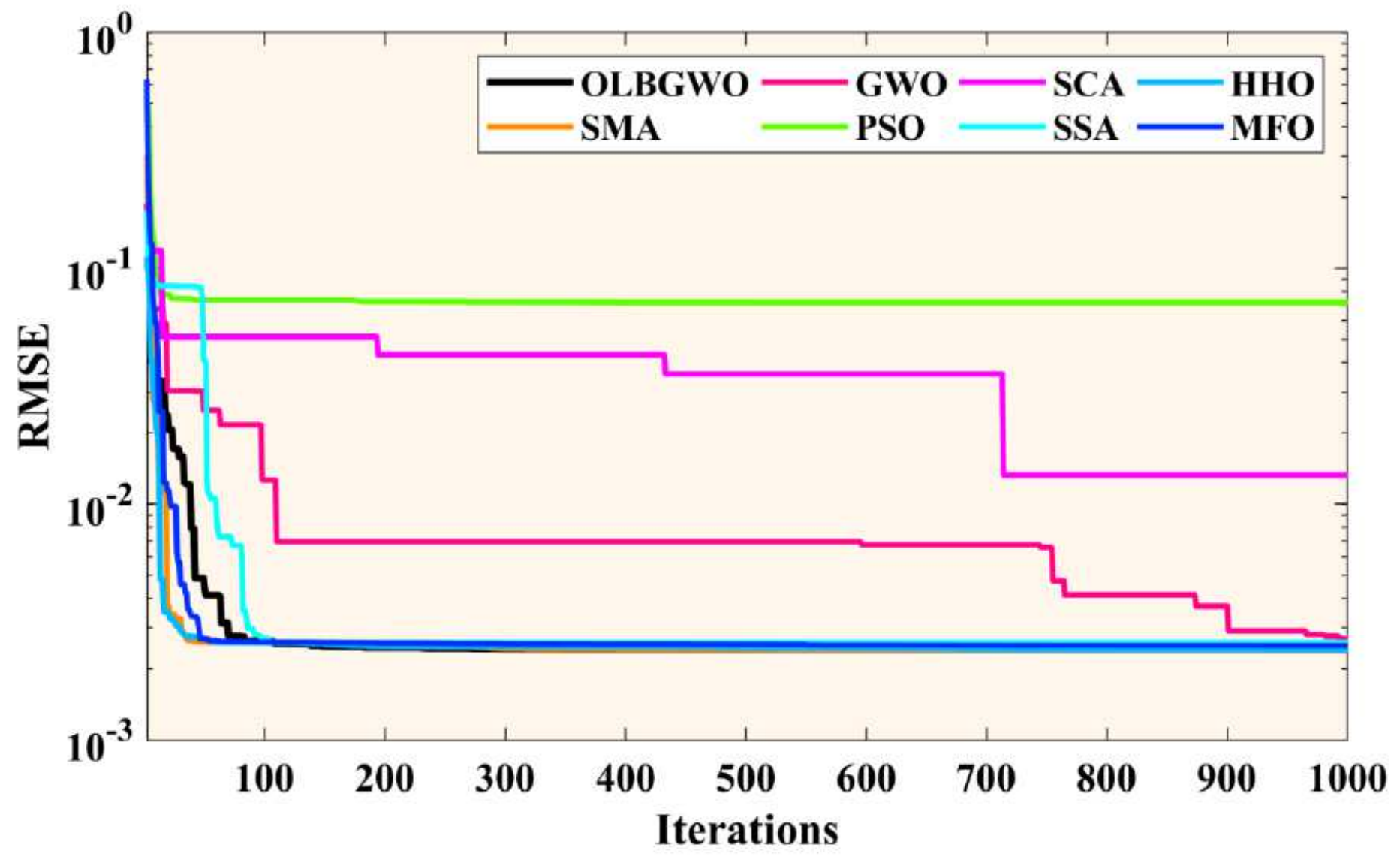

Figure 12

Convergence curve of all algorithms for the PV module model 




Figure 13

I-V characteristics of ST40 at $25^{\circ} \mathrm{C}$ and different irradiation 




Figure 14

$\mathrm{I}-\mathrm{V}$ characteristics of ST40 at $1000 \mathrm{~W} / \mathrm{m} 2$ and different temperature 


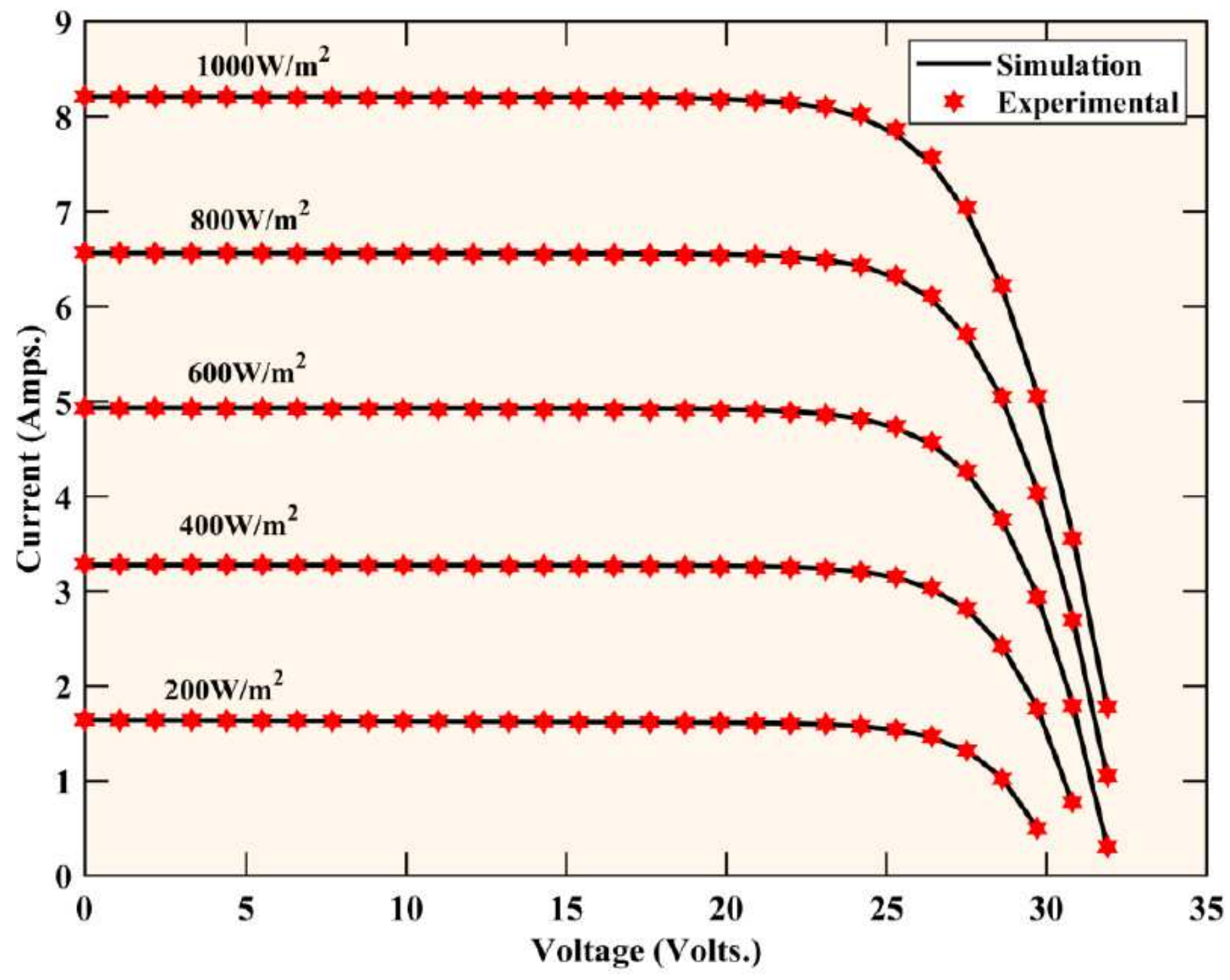

Figure 15

$\mathrm{I}-\mathrm{V}$ characteristics of $\mathrm{KC} 200 \mathrm{GT}$ at $25^{\circ} \mathrm{C}$ and different irradiation 


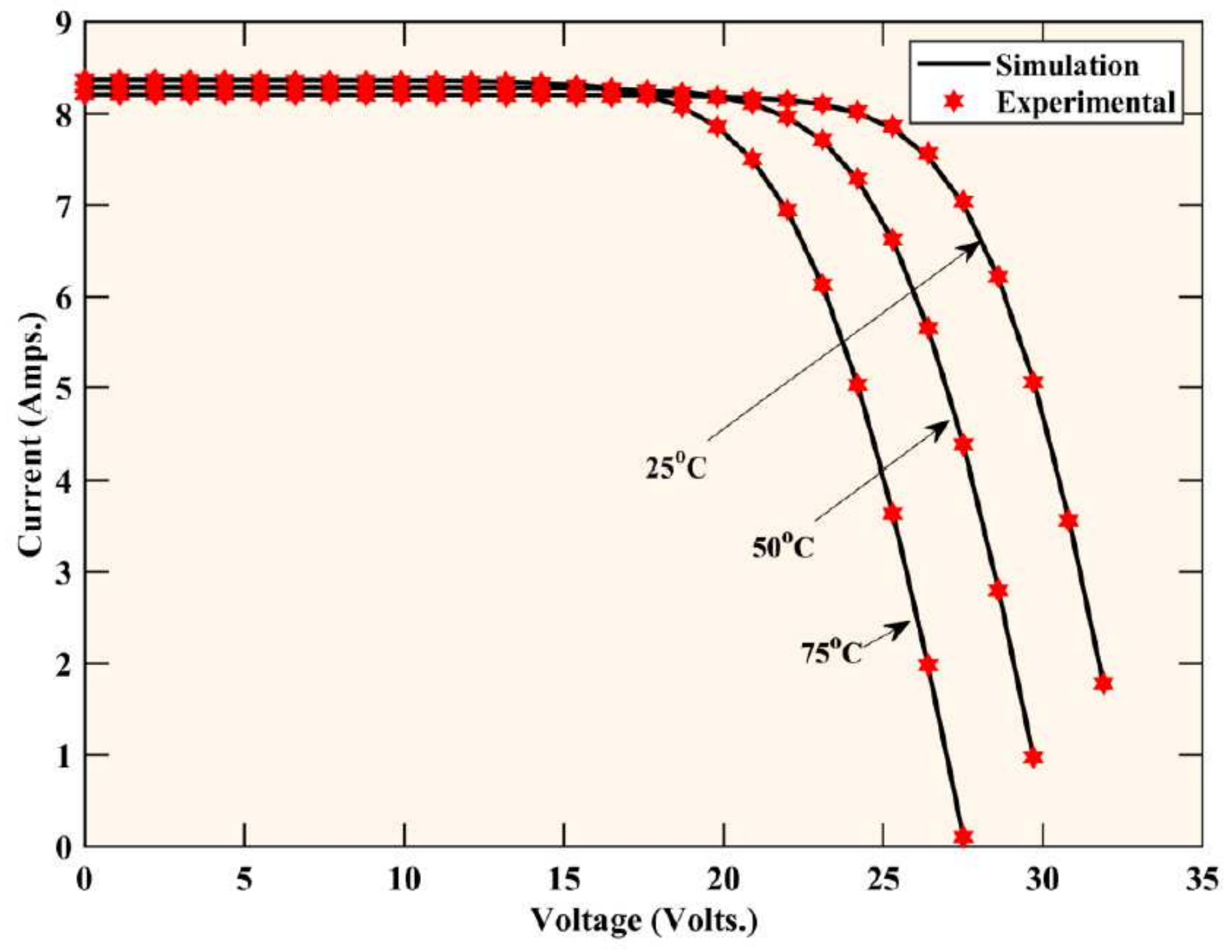

Figure 16

$\mathrm{I}-\mathrm{V}$ curves of KC200GT at $1000 \mathrm{~W} / \mathrm{m} 2$ and different temperature 